\title{
System-based DNA microarray analyses of different gene expression profile in spontaneously hypertensive rats treated with Songling Xuemaikang Capsule reveals underlying causal mechanisms.
}

\section{Li-tao Liu}

Chengdu Kanghong Pharmaceutical Group Co., Ltd. https://orcid.org/0000-0003-1199-2001

\section{Cui-qi Yan}

Chengdu Kanghong Pharmaceutical Group Co. Ltd.

\section{Qiao-xin Tang}

Chengdu Kanghong Pharmaceutical Group Co. LTD.

\section{Man-xi Zhao}

Chengdu Kanghong Pharmaceutical Group Co. Ltd.

\section{Chuan-zhen Teng}

Chengdu Kanghong Pharmaceutical Group Co. Ltd.

\section{Liang Ye}

Chengdu Kanghong Pharmaceutical Group Co. Ltd.

Xiao Ke ( $\nabla$ txzz@cnkh.com)

https://orcid.org/0000-0002-8714-2639

\section{Research article}

Keywords: Spontaneously hypertensive rats, Songling Xuemaikang Capsule, Gene expression profiling, Causal network

Posted Date: September 25th, 2019

DOI: https://doi.org/10.21203/rs.2.14612/v1

License: (c) (i) This work is licensed under a Creative Commons Attribution 4.0 International License. Read Full License 


\section{Abstract}

Background: Hypertension is considered the major risk factor for human health in the world. Songling Xuemaikang Capsule (SXC) is clinically used as a medicine for the prevention and treatment of cardiovascular and cerebrovascular diseases such as hypertension and hyperlipidemia. However, the underlying mechanisms have yet to be fully identified. Methods: Valsartan, as a positive control drug, high- and low-dose of SXC were orally administration with for 28 days to investigate the anti-hypertensive effect of SXC in spontaneously hypertensive rats (SHRs). The serum levels of aldosterone and Angiotensin II (Ang II) were detected. The gene expression profiling was performed in the thoracic aorta of SHRs using the Whole Rat Genome Oligo nucleotide Microarray. The integrated causal network analysis was performed to understand the mechanism of antihypertensive effect of SXC. Results: The results shown that the systolic and diastolic blood pressure were significant decreased in SXC low-dosage group and high-dosage group compared with the control group respectively. SXC low and high-dosage treatment decreased serum aldosterone levels significantly but increased serum Ang II compared with the control group respectively. Causal network analysis shown that treatment with SXC reversing the vascular remodeling process, inhibiting vascular inflammation and atherosclerosis, reversing endothelial cells dysfunction and likely reducing peripheral vascular resistance by down-regulated processes related to vascular remodeling, dyslipidemia, the complement system, leukocyte rolling, and endothelial dysfunction. In addition, SXC treatment may also activate fibrinolysis and regulate lipid and glucose metabolism. Conclusions: Those obtained data could help our understanding and potential utilization of SXC in the treatment or prevention of hypertension!

\section{Introduction}

Hypertension, a critical public health problem worldwide, is considered the major risk factor for ischemic and hemorrhagic stroke, myocardial infarction, heart failure, chronic kidney disease, peripheral vascular disease (PVD), cognitive decline, and premature death. Hypertension is the leading modifiable risk-factor for cardiovascular disease, which represents the top cause of death in China ${ }^{1,2}$. Hypertensive patients' blood pressure (BP) needs be controlled, to decrease the incidence of adverse sequelae. In China, the weighted prevalence of hypertension has been rising, with rates increasing from $18 \%$ in 2002 to $23.2 \%$ in $2015^{3,4}$. Among those with hypertension, $46.9 \%$ were aware of their condition, $40.7 \%$ were taking prescribed medication to lower their BP, but only $15.3 \%$ achieved BP control ${ }^{4}$.

Conventional antihypertensive agents ranging from diuretics, angiotensin-converting enzyme (ACE) inhibitors, angiotensin receptors blockers, sympathoplegic agents, calcium channel blocker, $\alpha$-adrenergic blockers and $\beta$ adrenergic blockers others are performed to control blood pressure levels in hypertensive patients ${ }^{5}$. Those agents are usually associated with many adverse effects such as hypokalemia, hyponatremia, severe dry cough, ankle edema, headache, facial flushing, polyuria and allergy. Traditional medicine is widely used, and is of increasing health and economic importance and often termed alternative or complementary medicine in many countries ${ }^{6}$. In some countries, the majority of the population continue to use traditional medicine to meet their health needs. This may, in part, be attributed to increasing concerns about the safety and approaches of Western medicine ${ }^{6}$. Overall, this may explain the increasing interest in panning out the beneficial health effects of various plants and herbs in different diseases including hypertension ${ }^{7}$.

Songling Xuemaikang Capsule (SXC) is a traditional Chinese patent medicine which has been authorized recommended by Chinese Pharmacopoeia. Three widely used traditional herbal medicines, Puerariae Lobatae 
Radix., Pine needles (Pinus densiflora Lamb.), and powdered nacre are containing in SXC. Puerarin extracted from Puerariae Lobatae Radix. possesses effects of antihypertension and stroke prevention by improved

microcirculation in spontaneously hypertensive rats (SHRs) through increasing in cerebral blood perfusion both by arteriole relaxation and $\mathrm{p} 42 / 44 \mathrm{MAPKs}$-mediated angiogenesis ${ }^{8}$ and protecting against endothelial dysfunction and end organ damage by anti-oxidant and upregulation of phosphor-eNOS ${ }^{9}$. Some components extracted from pine needle have been proved to have vasorelaxant effects by blocking the voltage-operated $\mathrm{Ca}^{2+}$ channel (VOCC) and inhibiting $\mathrm{Ca}^{2+}$ influx to the cytoplasmic ${ }^{10}$ and play an important role in the prevention of oxidative damage in vascular endothelial cells in hypertension patients via the PI3K/Akt/Bad signaling pathway ${ }^{11}$. Powdered nacre, mother of pearl, from Pinctada maxima, was showed the effect of reducing body weight, visceral fat amount, and blood triglyceride level without influencing the food intake, body length, or amount of muscular tissue ${ }^{12}$.

SXC has been widely used to treat hypertension and hypertension related symptoms in China. Preliminary clinical studies demonstrated that SXC could contribute to BP control and slow the progression of end-organ damage in hypertension ${ }^{13-16}$.

However, the molecular mechanisms involved in SXC treated hypertension remain largely undefined. Therefore, this study was carried out to investigate the antihypertensive effect of SXC in SHRs established animal model of essential hypertension. Furthermore, to elucidate its underlying mechanism, DNA microarray analyses were performed to obtain gene expression profiles in thoracic aorta tissue of SHRs after repeated oral administration of high- or low-dose of SXC and valsartan.

The aim of this study was to identify target genes which expression were markedly changed after oral administration of SXC in an established animal model of essential hypertension, and reconstructed a causal network to understand the molecular mechanism of in vivo antihypertensive activities of SXC.

\section{Methods}

\section{SHRs and Measurement of BP}

All the experiments were conducted in accordance with the National Institutes of Health Guide for the Care and Use of Laboratory Animals (NIH publication 85-23, revised 1996), and the experimental protocols were approved by the Institutional Animal Care and Use Committee (IACUC) of WuXi AppTec (Protocol Number: 27-2956). All surgery was performed under sodium pentobarbital anesthesia, and all efforts were made to minimize suffering.

SHRs (8-10 weeks old, male, SPF, 280-330 g body weight) were purchased from Beijing Vital River Laboratory Animal Technology Co., Ltd. (Beijing, China). The animals are kept in an animal breeding room where the environmental conditions are strictly controlled. The temperature in the rearing room is maintained at 20 to $24^{\circ} \mathrm{C}$, and the humidity is maintained at 30 to $70 \%$. The daylighting of the animal husbandry is controlled by an electronic timed lighting system that turns off the lights for 12 hours every day and on for 12 hours. During the experiment, the animals were kept in single cages. They were fed with standard laboratory diet and guaranteed free drinking water.

The rats were acclimatized in the above conditions for about a week before the experiment. Compound anesthesia was performed with $3 \%$ sodium pentobarbital solution $\left(60 \mathrm{mg}^{\circ} \mathrm{kg}^{-1}\right.$, i.p.). HD-S10 Small Animal Implant (Data Sciences International, New Brighton, MN, USA) was implanted on Day-3 Day-4. The procedure was as follows: 
the incision was performed on the abdomen of the rat. The catheter of the implant was inserted into the separated abdominal aorta. Bio-gel was used to stop bleeding. The implant was fixed on the abdominal wall. Then, the muscle and skin were sutured and sterilized. Subcutaneous injection of ibuprofen for analgesia. Gentamicin (8 $\mathrm{mg} \cdot \mathrm{kg}^{-1} \cdot \mathrm{day}^{-1}$, i.p.) was intraperitoneally administered three days after surgery to prevent infection.

SHRs were randomly divided into three groups: model group $(n=8)$, rats were orally administered with saline $\left(7.5 \mathrm{~mL} \cdot \mathrm{kg}^{-1}\right)$ for 4 weeks; SXC low-dose group $(\mathrm{n}=6)$, rats were orally administered with SXC $\left(236.25 \mathrm{mg} \cdot \mathrm{kg}^{-1}\right)$ for 4 weeks; SXC high-dose group ( $n=6)$, rats were orally administered with SXC $\left(708.75 \mathrm{mg}^{\circ} \mathrm{kg}^{-1}\right)$ for 4 weeks; the positive group $(n=6)$, rats were orally administered with valsartan $\left(21.6 \mathrm{mg}^{\circ} \mathrm{kg}^{-1}\right)$ for 4 weeks. The SXC was orally administered at 9:00-10:00 and 12:00-13:00 every day and the valsartan was administered at 14:00-15:00 every day. The BP of each rat was measured at 15:00-17:00 on every Thursday via implantable telemetry technology with Dataquest ART system (Data Sciences International., New Brighton, MN, USA). Ten BP readings were obtained for each rat and averaged.

\section{Isolation of Animal Tissue}

At 28 days, all rats were killed with intraperitoneal injection of $3 \%$ sodium pentobarbital solution $\left(60 \mathrm{mg}^{\circ} \mathrm{kg}^{-1}\right)$, and $3 \mathrm{~mL}$ blood samples were collected from the heart. These blood samples were transferred immediately into aseptic capped tubes and centrifuged at $3000 \mathrm{~g}$ for $4 \mathrm{~min}$ at $4^{\circ} \mathrm{C}$. The plasma supernatant was collected and stored at $80^{\circ} \mathrm{C}$ until further analysis. The thoracic aorta dissected from each rat was shredded and immediately stored in liquid nitrogen. The thoracic aorta of all animals was collected for DNA microarray analyses from each group. The levels of Angiotensin II (Ang II), Aldosterone in serum of SHRs from different groups were separately determined by the enzyme-linked immunoassay kit (Mybiosource, San Diego, CA, USA).

\section{DNA Microarray Analysis}

The following procedures for sample preparation and microarray analysis were done at Agilent Whole Genome Oligo Microarrays (Agilent, Santa Clara, CA, USA). Total RNA was extracted and purified from thoracic aorta tissue separately by TRIzol ${ }^{\circledR}$ Reagent (Invitrogen life technologies, Carlsbad, CA, USA) and Cleaned-up by RNasey Mini Kit (Qiagen, Valencia, CA, USA). Then, the Cy3-labeled cRNA was transcribed from $1 \mu \mathrm{g}$ of RNA of each group using the Quick Amp Labeling Kit, One-Color (Agilent, Santa Clara, CA, USA). Cy3-labeled cRNA was hybridized to the Whole Rat Genome Oligonucleotide Microarray ver. 3.0 (4×44k, G2505C-028282) (Agilent Technologies), following the manufacturer's hybridization protocol. After the washing step, the microarray slides were scanned using Agilent Microarray Scanner (Agilent G2505C, Agilent Technologies, Santa Clara, CA, USA) according to the manufacture's protocol. Microarray expression data were analyzed using Agilent Feature Extraction software (Agilent Technologies, Santa Clara, CA, USA) with the default settings for all parameters. The raw data were firstly normalized with the quantile algorithm, and the probes that at least one out of all samples had fluorescence signals in detection were chosen for further analyses.

\section{Pre-processing of microarray data and identification of differential expression genes}


The microarray data was processed using the $R$ statistical language ( $R$ version 3.4.2) and the limma package ${ }^{17}$. Beginning from the raw Agilent data files, we background-corrected and normalized between arrays using the quantile normalization method ${ }^{18}$. The normalized log2 intensities were visualized using principal components analysis (PCA) and hierarchical clustering. Limma package was used to performed the differential expression analysis. The differential expression genes (DEGs) fold-changes, P-values, and adjusted P-values were calculated for multiple testing by using the Benjamini-Hochberg method ${ }^{19}$. Gene lists filtered for padj (adjusted $p$-value) < 0.01 were used for further analysis.

\section{Gene ontology and pathway enrichment analyses}

For those genes which were differently expressed among group, enrichment analysis was performed by using a hypergeometric test to compare the gene list with several functional ontologies available in MetaBase (Thomsan Reuters, New York, NY, USA). The ontologies of interest are Clarivate Pathway Maps, Gene Ontology (GO) Biological Processes and GO Molecular Functions. The significance of enrichment of the list genes in functional ontologies in MetaCore (Thomsan Reuters, New York, NY, USA) is defined by P-values of hypergeometric distribution.

\section{Integration of Causal Network Model analysis}

The topologically significant genes (TSGs) were identified by overconnection, hidden nodes, and causal reasoning tests. An overconnectivity test ${ }^{20,21}$ identifies regulators of a DEG list that are directly connected and statistically overconnected to these DEGs. Hidden nodes analysis, the second network analysis method, is applied to identify genes with a high level of connectivity within an unlimited number of steps away from the data. The score is calculated using a conditionspecific network that joins all of the differentially expressed genes together via shortest paths ${ }^{21,22}$. Causal Reasoning is a shortest-path search based method which predicts upstream regulators causal for gene expression changes observed in experimental data ${ }^{23}$. The method prioritizes nodes in a molecular network that are likely responsible for the observed modulations in an experiment.

A hypergeometric test was performed for the gene list of DEGs and TSGs for enrichment analysis. Pathway maps simultaneously enriched with both the DEGs and the corresponding TSGs $(P<0.05$. The pathways from the step above are tested for "synergistic" behavior of the DEGs and the TSGs with enrichment synergy method ${ }^{24}$. Then, the finale key pathways are those which are enriched not only with DEGs but also with their regulators and where these two enrichments are synergistic. All the causal networks were constructed through MetaCore (Thomsan Reuters, New York, NY, USA)

\section{Statistical analysis}

Data was analyzed using GraphPad Prism 5.0 software (GraphPad Software, Inc., La Jolla, CA, USA). One-way analysis of variance followed by Tukey's post hoc test was used for comparisons among multiple groups. A value of $\mathrm{P}<0.05$ was considered statistically significant.

\section{Results}

\section{SXC can lower blood pressure in SHRs}


Systolic BP and diastolic BP of SHRs were measured on every Thursday, at 2 hours after SXC treatment and valsartan for 4 weeks before DNA microarray analyses. As the results shown in table 1, both the systolic BP and diastolic BP were well controlled both in SXC high- and low-dose groups from the beginning to the end of experiment. Valsartan treated group dramatic decreased the systolic BP and diastolic BP from the $1^{\text {st }}$ week (Table 1). While compared to the rats in the control group, the systolic BP and diastolic BP were significant decreased in the rats of SXC high- and low-dose groups from the $3^{\text {rd }}$ week (Table 1). At the end of the experiment, the systolic BP and diastolic BP were significant decreased by $13.50 \%, 29.57 \%$ in SXC low-dosage group and $6.82 \%, 26.82 \%$ in SXC high-dosage group compared to the control group respectively (Table 1). These results demonstrated SXC could be potentially used in the treatment of hypertension.

\section{SXC reduces serum aldosterone content}

The content of Angll and aldosterone in serum were detected via ELISA. Valsartan and two dosage SXC treatment all increased serum Ang II levels (Fig 1A). As the results shown in Fig 1B, the serum aldosterone levels were significantly decreased by $12.66 \%$ and $41.86 \%$ in SXC low and high-dosage administrated groups compared with the control group respectively, while those in valsartan group was increased by $74.03 \%$ compared with the control group.

\section{Identification and Hierarchical Clustering of DEGs}

We used 44K gene chip microarray to identify differentially expressed genes in the thoracic aorta of SHRs treated with SXC low-dose, SXC high-dose and valsartan compared with control SHRs respectively. Limma was used to calculate fold-changes, P-values, and adjusted P-values for each comparison. The PCA plot of all samples shows much more robust clustering by sample group (Fig 2A). The volcano plots shown below in summarize the corrected p-values and log2 fold-changes that were calculated for each treatment (Fig 2B-D). Compared with model group, there are 6403 (2688 up-regulated \& 3715 down-regulated), 5144 (2783 up-regulated \& 2361 down-regulated) and 6285 (2760 up-regulated \& 3525 down-regulated) genes altered expression in valsartan group, SXC low-dose group and SXC high-dose group respectively (FDR<0.05). These altered expression genes represented 1133 (364 upregulated \& 769 down-regulated), 706 (548 up-regulated \& 158 down-regulated) and 920 (367 up-regulated \& 553 down-regulated) unique genes in valsartan group, SXC low-dose group and SXC high-dose group respectively. When the fold change threshold was set as 2.0, a total of 2790 (1218 up-regulated \& 1572 down-regulated), 1679 (893 upregulated \& 786 down-regulated) and 2600 (1168 up-regulated \& 1432 down-regulated) genes were identified as DEGs in valsartan group, SXC low-dose group and SXC high-dose group compared with the control rats respectively (FDR<0.05). Additional information on all DEGs is listed in Table S1-S3. The rank top 500 DEGs in a heatmap was shown in Fig. 3. Their expression patterns presented similar functions, suggesting that the DEGs were clustered. The results precisely distinguished the DEGs of drug treated samples (valsartan, SXC low-dose and SXC high-dose) and the control group. Moreover, the expression patterns of the DEGs were predominantly downregulated in the SXC treated samples.

\section{Enrichment analysis}

GO analysis was performed on for the top 500 up-regulated and down-regulated DEGs ranked by FDR. This analysis consists of three parts: GO biological processes, GO molecular functions, GO cellular compartments. Top 5 
GO enrichment terms in every category were selected and shown in Fig 4. We found the frequency of downregulated genes were involved in GO cellular compartments terms related to the extracellular region which highly overlapped between the three treatments. Synapse, up-regulated GO cellular compartment was commonly enriched in valsartan and SXC high-dose treated groups. Up-regulated GO cellular compartments potassium channel complex was mostly enriched only when treated with valsartan while high-density lipoprotein particle was enriched only when treated SXC low-dose. For the biological process, the enriched down-regulated GO Biological processes included metabolic processes and leukocyte mediated immunity.

When treated with SXC high-dose and valsartan synaptic signaling was the up-regulated GO biological processes. Regulation of Wnt signaling was the up-regulated GO biological processes only in SXC high-dose treated group. The down-regulated GO molecular functions were significantly ascribed to oxidoreductase activity, likely related to lipid metabolism in all the three treatment. The up-regulated GO molecular functions were primarily ascribed to gated channel activity in SXC high-dose and valsartan treated groups, but mostly enriched in molecular function regulator in SXC low-dose treated group.

After the Go enrichment analysis, to overview the function of differential expressed genes in three treatment, these DEGs were utilized in the focused analysis to identify the Clarivate pathways in the three treatments compared to the control group. These results are shown in Fig 5. The up-regulated enriched Clarivate pathway maps were not significant after multiple comparison correction. The top 5 up-regulated Clarivate pathway maps were related to cardiac myogenesis in SXC high-dose and valsartan treated groups. The top 5 down-regulated Clarivate pathway maps were more ascribed in Peroxisome proliferator-activated receptors (PPAR) regulation of lipid metabolism and complement system induced immune response in valsartan treated group. In SXC high-dose and low dose treated group, the top 5 down-regulated Clarivate pathways were related to PPAR regulation of lipid metabolism, complement system induced immune response, inflammation and vascular and endothelial dysfunction.

\section{Identification of topologically significant genes}

To identify topologically significant genes (Ts genes), network-based analyses including: overconnectivity test, hidden nodes analysis, and causal reasoning analysis were performed. The overconnected nodes, acted as upstream regulators of differential expression were tested in each sample group independently by an overconnectivity test which identifies regulators of a DEGs list that are directly connected and statistically overconnected to these DEGs. As shown in Table 2, SP1 is the top ranked overconnected gene for all treatments, which involved in many cellular processes, including cell growth and differentiation, apoptosis, immune responses, DNA damage response, and chromatin remodeling ${ }^{25}$. PPAR-alpha and PPAR-gamma, and HIF1A were also identified as the overconnected transcription factors for all the three treatment. PPARs, a steroid hormone receptor, is a member of the nuclear receptor superfamily. Isomers of PPARs include PPAR-alpha, PPAR-beta, and PPARgamma, regulating metabolic processes including lipid and glucose homeostasis, and inflammatory responses ${ }^{26-}$ ${ }^{28}$.HIF1A, a master transcriptional regulator of the adaptive response to hypoxia, has a key role in cellular response to hypoxia, including the regulation of genes involved in energy metabolism, vascular remodeling, and atherosclerosis ${ }^{29}$.

Hidden nodes analysis is based on shortest-path method that identifies genes with a high level of connectivity within an unlimited number of steps away from the data. Highly significant genes from this analysis are likely to be functionally involved in the molecular changes, including a driver role, despite not being differentially expressed at 
the transcriptional level themselves. PLAUR (UPAR) was identified as the top hidden node for both doses of SXC treatments. This gene codes for the plasminogen activator, which is involved in blood coagulation, cell adhesion, and inflammation via the JAK-STAT pathway. Fibronectin, ADAM12, and c-Fos were also highly ranked hidden nodes for both doses of SXC treatments. Fibronectin and ADAM12 are both involved in cell adhesion and extracellular matrix, while c-Fos is a transcription factor that participates in many signaling transduction cascades, including some related to inflammation and immune response (Table 2).

Causal reasoning analyses, another shortest-path search-based method, predicts upstream regulators causal for gene expression changes observed in experimental data. Causal reasoning analyses prioritizes nodes in a molecular network that are likely responsible for the observed modulations in an experiment and takes the directionality of the edges into account as well as the biological effects (activation or inhibition).

CNOT3 was a top-ranked causal reasoning regulator for all three treatments. This gene is a general transcription regulator and is involved in bulk mRNA degradation, miRNA-mediated expression, and translational repression 30 (Table 2).

\section{Causal network models demonstrated the molecular mechanism of SXC for control blood pressure}

While DEGs and functional pathways affected in the thoracic aortas of SHRs with different treatment were identified, the complex nature of hypertension makes it impossible to elucidate whether these changes are directly contributed to the therapeutics treatment or simply a reflection of individual differences or loss of critical regulatory signals. To our best knowledge, this is the first attempt applying causal network models to analyses transcriptional changes present in the therapeutics treatment of hypertension thoracic aortas tissues. To shed light on the causal processes underlying the expression changes observed in SHRs thoracic aortas regions, affected genes which either differentially expressed or topologically significant were searched among triggers of the signaling pathways on the key maps. Next, signal transduction pathways were selected that were downstream of affected triggers and contained consecutive signaling molecules belonging to affected genes. The identified pathways down to the topologically significant transcription factors (TFs) and/or cellular functional effects were traced and shown on the map. Causal models reconstructed with such a procedure illuminate mechanisms linking the different levels of cell regulation into a meaningful integrated system and provide a roadmap to the relevant signaling mechanisms. As shown in Table 3, the top 10 enriched key maps in the SXC high dose compared to control group involve mostly immune response, cell adhesion, vascular damage and lipid metabolism processes. These maps, along with others including enriched pathway maps and maps related to vascular cells and smooth muscle contraction were used to reconstruct a causal network model. The network model is divided into 2 maps (Fig 6 A and B). Several functional areas are highlighted on the maps: Lipid metabolism, Dyslipidemia and Inflammation, Complement System and Immune Response, Leukocyte Rolling and Endothelial Dysfunction and Vascular Remodeling. As shown in Table 4, the top 10 enriched key maps in the valsartan treated group compared to control group involve mostly in lipid metabolism processes. These maps, along with others including enriched pathway maps and maps related to Gprotein coupled receptor signaling cascades, were used to reconstruct a causal network model. The network model is divided into 2 maps (Fig 7 A and B). Several functional areas are highlighted on the maps: Vascular remodeling, smooth muscle relaxation and lipid metabolism.

\section{The comparison of key pathways different therapy treatment}


In order to identify the difference between SXC high-dose treatment and valsartan treatment in SHRs, all the dysregulated key pathways in both two treatment were compared. In the analysis of key pathway maps, 16 maps were found to be shared between the SXC high-dose and valsarta treatments. These key pathway maps are shown below in Table 5, with the p-values from the hypergeometric test performed. The overlapping maps describe processes related to lipid metabolism and dyslipidemia, endothelial dysfunction, inflammation, and platelet activation. All processes seem equally distributed between the two drugs, except for endothelial dysfunction such as role of cell adhesion in vaso-occlusion in sickle cell disease and vascular endothelial cell damage in systemic lupus erythematosus (SLE), which perhaps shows a small trend toward being more enriched in SXC treatment. In both groups, the treatment down-regulates the immune response and associated inflammatory processes, as well as dyslipidemia and lipid metabolism processes. Both treatments also play a role in reversing the effects of the vascular remodeling that takes places during hypertension.

There are 30 key pathways that are unique to the SXC high dose group are shown below in Table 6. These maps relate mostly to the immune response triggered by complement system, inflammatory cytokines, and detailed pathway maps involving platelet activation, leukocyte rolling and cell adhesion processes that play a role in vascular remodeling and cardiovascular disorders. In the case of valsartan treated groups, the 31 unique key maps, shown below in Table 7, relate mostly to the angiotensin system, further dyslipidemia, lipid-related maps and oxidative stress.

\section{Discussion}

Elucidating the mechanism of action of drugs especially the Traditional Chinese medicine (TCM) and the causal events after drug treatment are challenging, but they are one of the primary goals of medical research.

Understanding the mechanism of action of a drug can prioritize drug candidates or help identify novel indications for existing drugs. Because the direct target of the SXC treatment for hypertension is unknown, understanding the physiological effects of the treatment provides further insights into disease pathways and how they can be rescued. Microarrays provides high-resolution data that can reveal much about the state of the cell, tissue, and organism during treatment. Clarivate Analytics performed differential expression analysis on microarray data obtained from thoracic aorta samples in SHR rats treated with valsartan, SXC high-dose, SXC low-dose or appropriate vehicle controls.

The microarray data was high quality and thousands of differentially expressed genes were identified in each drugcontrol comparison. There was a high degree of overlap in gene expression, both between valsartan and SXC highdose, and the two SXC dosages. GO analysis and the Clarivate pathways were performed to overview the function of differential expressed genes in three treatment. The up-regulated ontologies were not significant after corrected multiple testing for $\mathrm{GO}$ cellular compartments, $\mathrm{GO}$ biological processes and $\mathrm{GO}$ molecular functions. The downregulated ontologies were significantly ascribed to oxidoreductase activity, likely related to lipid metabolism for GO molecular functions, metabolic processes and leukocyte mediated immunity for GO biological processes and extracellular region for GO cellular compartments in all the three treatment. The up-regulated enriched Clarivate pathway maps were also not significant after multiple comparison correction. The down-regulated Clarivate pathway maps were more ascribed in PPAR regulation of lipid metabolism and immune response in valsartan treated group. The previous studies demonstrated valsartan therapy significantly lowered total- and LDL-cholesterol levels and had significant anti-inflammatory efficacy in hypertensive diabetic patients with enhanced inflammatory burden $^{31,32}$. The enrichment analysis indicated that SXC treated SHRs down-regulated Clarivate pathways not only 
including PPAR regulation of lipid metabolism, complement system induced immune response but also including inflammation and vascular and endothelial dysfunction in SXC high-dose and low dose treated group.

Additionally, common transcript changes with different treatment were assessed using causal network analyses. Mechanism reconstruction through causal network models provides an integrated view of how a drug effects the cell and insight into previously unrecognized therapeutic mechanisms of the drug. In-depth investigation revealed several common molecular drivers down-regulated in both SXC high-dose treated group and valsartan treated group. These changes were integrated and comprised our causal map. The causal maps reflected some functional areas were shared between SXC treatment and valsartan treatment but through differing pathways in each treatment. Those common functional areas including "lipid metabolism, dyslipidemia and inflammation" and "Vascular remodeling" are detailed below.

Multiple cohort studies have strongly indicated a causal relationship between dyslipidemia and risk of developing hypertension. One possible explanation for this relationship is that hypertension and dyslipidemia share common pathophysiological etiologies, such as obesity and the resulting dysregulation of adipocytokine release from adipose tissue. Furthermore, dyslipidemia adversely affects functional and structural arterial properties and promotes atherosclerosis. These changes may impair BP regulation, which, in turn, predisposes individuals with dyslipidemia to development of hypertension ${ }^{33}$. AGTR1 mediates the predominant actions of angiotensin II on blood pressure, and it is the target of a widely used class of antihypertensive drugs: the angiotensin receptor antagonists $^{34}$. This is in line with multiple studies which show an association between AGTR1 blocker treatment and downregulated lipid metabolism ${ }^{35-37}$. In our results, lipid metabolism, dyslipidemia and inflammation are down-regulated processes both upon treatment with valsartan and high dose SXC. HIF1A and PPAR-gamma appear to act as transcriptional regulators of the process which both down-regulated in both two treatment. Angiotensin II, acting through AGTR1, increases HIF-1A nuclear localization and activity. However, AGTR1 is downregulated in valsartan treated group, thereby down-regulating HIF-1A and its downstream effects on lipid metabolism. Both two treatment down-regulated LPL, VLDLR, ABCG1 and ABCA1 which lead to decreased dyslipidemia. Low levels of adiponectin are associated with endothelial dysfunction, hypertension and cardiovascular disease ${ }^{38-40}$. Curiously, adiponectin is downregulated in both two treatment but with different regulators. Downregulated HIF-1A is the main influence factor of down-regulated adiponectin which also leads to downregulated PPCKC playing a role in gluconeogenesis in treatment with valsartan. It seems that more than one regulator changed caused adiponectin down-regulated when treated with SXC high dose. It is possible that this is explained by the fact that treatment with high dose SXC causes downregulation of BMP4, which through SMAD and PPAR-gamma regulates the transcription of A-FABP, LPL, Perilipin, CIDEC and Factor D which down-regulated contributing for decreased expression and differentiation of white fat genes and release of adiponectin.by downregulated HIF-1A. Additional, high dose SXC treatment also downregulated perilipin, PGAR and PPARGC1 by decreasing lipolysis. Upon SXC high dose treatment, HIF1A also down-regulates the expression of various genes that are known to play a role in inflammation: IL-1 beta, PAI-1, PBEF and IL -6 , the latter doing so through the JAK/STAT and ERK/MEK cascades. Heme oxygenase 1, however, was also downregulated.

Vascular remodeling is an active process of structural modification that involves changes in at least four cellular processes: cell growth, cell death, cell migration, and the synthesis or degradation of extracellular matrix. Vascular remodeling is dependent on dynamic interactions between local growth factors, vasoactive substances, and hemodynamic stimuli and is an active process that occurs in response to long-standing changes in hemodynamic 
conditions; however, it may subsequently contribute to the pathophysiology of vascular diseases and circulatory disorders. Likewise, vascular fibrosis entails accumulation of collagen, fibronectin, and another extracellular matrix

components in the vessel wall and is an important aspect of extracellular matrix remodeling in hypertension ${ }^{41,42}$. Treatment with valsartan and high dose SXC appears to induce changes in vascular remodeling through different regulatory pathways. Actomyosin, responsible for cytoskeleton remodeling and regulation of smooth muscle contraction, is highly upregulated via MRLC upon both treatment. In treatment with valsartan, downregulated AGTR1 caused a series of $G$ protein-coupled receptors (GPCRs) regulated vascular tone was down-regulated which up-regulated actomyosin through Gq/G11/LARG/RhoA/ROCK/MRLV pathway, whereas in treatment with high dose SXC actomyosin was up-regulated by RhoA/ROCK/MRLV and Rac/PAK/MRLV pathways. Phosphorylation of myosin light chains by MLCK triggers cross-bridge cycling between actin (downregulated) and myosin (upregulated) filaments ${ }^{43}$. Down-regulation of PLC leads to a decrease in IP3 production, which reduces the release of calcium ions from the endoplasmic reticulum into the cytoplasm reducing the expression of calmodulin and thus reducing the activation of MLCK in treatment with valsartan. Likewise, several channel pumps (TRPC1, NCX1 and MaxiK) are deregulated upon treatment with valsartan, generating an exchange of calcium, sodium and potassium ions between the various compartments and contributing to smooth muscle contraction regulation. Hypertension-induced mitochondrial structural abnormalities are often accompanied by alterations in mitochondrial metabolic and bioenergetic functions. Valsartan also downregulated mitochondrial biogenesis by down-regulated PPAGC1 which played a central role in the regulation of mitochondrial biogenesis. It could be explained by the down-regulated eNOS and VEGF-A by valsartan ${ }^{44,45}$. Mitochondrial biogenesis and oxygen consumption increase markedly during adipogenic differentiation, and reducing mitochondrial respiration by hypoxia or by inhibition of the mitochondrial electron transport chain significantly suppresses adipogenic differentiation ${ }^{46}$. In treatment with high dose SXC, cytoskeletal actin is slightly downregulated. Treatment with high dose SXC also alters vascular remodeling through other pathways. In vascular structures some results suggest that actin polymerization increases as vasoconstriction is prolonged ${ }^{47}$. Treatment with high dose SXC also regulated IL-6 /JAK/STAT pathway which regulating the secretion of growth and migration factors like VEGF-A, Cathepsin B (both downregulated in this dataset), MMP-9 (regulator)/collagen and Stromelysin-2/Nidogen pathways which are involved in extracellular matrix and cytoskeleton remodeling. TGF-a release by ADAM-17 has also been implicated in hypertension-induced vascular remodeling in mouse carotid arteries ${ }^{48}$. Treatment with high dose SXC down-regulates $A D A M-17$, and there is evidence that ADAM-17 binds glycoprotein $\mathrm{VI}$ and triggers platelet aggregation and thrombus formation. Increased plasminogen activator inhibitor type 1 (PAI-1) levels and decreased tissue plasminogen activator activity resulting impaired fibrinolytic function have been found in patients with hypertension and may account in part for the increased risk of atherosclerosis ${ }^{49}$. Treatment with high dose SXC appears to activate of fibrinolysis via downregulated PAI-1 and upregulated plasminogen and fibrinogen. Downregulated PAI- 1 also leaves thrombin to exert its fibrinolytic function via cleavage of fibrinogen in treatment of high dose SXC. Epithelial-to-mesenchymal transition (EMT) leading to fibrosis and vascular alterations appears to play a role in numerous chronic cardiovascular disease states such as heart failure, pulmonary hypertension and various forms of chronic vasculopathy ${ }^{50}$. Treatment with high dose SXC appears to down-regulate this process via IL-6/JAK/STAT pathway, N-cadherin and vimentin.

The mostly interesting finding in our study is that high dose of SXC treatment has more altered the complementmediated immune response in vascular and reduced the enrichment of lymphocytes in hypertensive injured vascular. Treatment with high dose of SXC causes downregulation of the complement system. Tissue injury, the primary signal for launching the innate immune response and inflammation, initiates several signaling cascades 
that regulate changes in the microvasculature, including the activation of complement. Complement activation may drive the pathology of hypertension and hypertensive injury through its impact on innate and adaptive immune responses. In addition to the proinflammatory properties of complement, complement cleavage fragments of C3 and C5 (the latter upregulated in this dataset) can exert anti-inflammatory effects that dampen the inflammatory response to injury. Complement components are engaged in the regulation of multiple phases of an inflammatory reaction, including changes in vascular flow, increase in vascular permeability, extravasation of leukocytes, and chemotaxis ${ }^{51,52}$. IL-6 and C-reactive protein (CRP) are regulators of the complement system. IL-6 produces inflammatory effects by inducing the transcription of factors in multiple pathways of inflammation such as JAK/STAT pathway, which is downregulated upon treatment with high dose of SXC. Treatment with high dose of SXC causes downregulation of the leukocyte rolling and ultimately intima hyperplasia and endothelial dysfunction. A wealth of evidence indicates a fundamental role for inflammation in the pathogenesis of cardiovascular disease (CVD), contributing to the development and progression of atherosclerotic lesion formation, plaque rupture, and thrombosis. An increasing body of evidence also supports a functional role for complement activation in the pathogenesis of CVD through pleiotropic effects on endothelial and hematopoietic cell function and hemostasis. Complement activation contributes to endothelial cell activation; leukocyte and vascular smooth muscle cell (VSMC) migration; platelet adhesion, activation and aggregation; activation of coagulation; and impaired fibrinolysis ${ }^{53,54}$. Treatment with high dose SXC appears to inhibit all these processes. In particular, platelet activation and aggregation are inhibited via C1q/PKC/P-selectin and collagen I/collagen III/FC gamma/RII alpha receptor. Also inhibited by treatment are platelet-neutrophil-endothelial cell aggregations within the vasculature, which are otherwise increased in CVD and even in risk factors such as hypertension and dyslipidemia. Binding of ICAM1 with alpha M/beta-2 integrin receptor (also downregulated in this dataset) permits interactions to occur between neutrophils and endothelial cells, which in turn cause leukocyte rolling, adhesion, and finally transmigration to the intima layer of the vasculature. Eosinophil-platelet adhesion, which occurs during thrombosis, is also downregulated.

Taken together we used SHRs to analyze the hypotensive effects of SXC and valsartan in vivo, and reported the overall gene expression pro-files from thoracic aorta of SHRs orally treated with SXC for the first time. Our results suggest that dietary intake of SXC had mild BP lowering effect. Both valsartan and SXC high-dose down-regulated processes related to vascular remodeling and dyslipidemia. Smooth muscle relaxation was unique key pathway to valsartan due to its action as an angiotensin receptor antagonist, while down-regulation of the complement system, leukocyte rolling, and endothelial dysfunction was unique key pathway to SXC. The effects of SXC high-dose treatment appear to be broader than valsartan and the benefits could be summarized as follows. SXC high-dose treatment reverses the vascular remodeling process, it inhibits vascular inflammation and atherosclerosis by inhibiting platelet activation, adhesion and activation, as well as adhesion between platelets, endothelial cells, and leukocytes, thus reversing endothelial dysfunction and likely reducing peripheral vascular resistance. It also appears to activate fibrinolysis thereby inhibiting thrombus formation. In addition, it regulates the metabolism of lipids and glucose, which are associated with multiple conditions such as obesity, diabetes, metabolic syndrome, and cardiovascular disorders.

\section{Conclusions}

Those results provided valuable information for our understanding of the molecular mechanisms that underlie the potential antihypertensive activities of SXC, and will contribute towards increased value-added utilization of SXC. 


\section{Abbreviations}

$S X C$, Songling Xuemaikang Capsule; SHRs, spontaneously hypertensive rats; $P V D$, peripheral vascular disease; $B P$, blood pressure; $A C E$, angiotensin-converting enzyme; Ang II, Angiotensin II; $P C A$, principal components analysis; $D E G s$, differential expression genes; GO, Gene Ontology; TSGs, topologically significant genes; PPARs, peroxisome proliferator-activated receptors; $T F s$, transcription factors; $S L E$, systemic lupus erythematosus; $T C M$, Traditional Chinese medicine; AGTR1, angiotensin receptor 1; HIF1A, hypoxia-inducible factor 1A; VLDLR, very low density lipoprotein receptor; $A B C G 1$, ATP binding cassette transporter G1; $A B C A 1$, ATP-binding cassette, subfamily A, member 1; $B M P 4$, bone morphogenetic protein $4 ; A-F A B P$, adipocyte fatty acid-binding protein; $P G A R$, peroxisome proliferator-activated receptor $\mathrm{y}$ angiopoietin-related gene; $P P A R G C 1$, peroxisome proliferator-activated receptor- $\mathrm{Y}$ co-activator-1; $P A I-1$, plasminogen activator inhibitor-1; $P B E F$, pre-B cell colony-enhancing factor; $I L-1$, Interleukin-1; IL-6, Interleukin-6; JAK/STAT, Janus kinase-signal transducer and activator of transcription; $E R K$, extracellular signal-regulated kinase; $M A P K$, mitogen-activated protein kinase; $M R L C$, multi resolution land characteristics; $L A R G$, leukemia-associated Rho GEF; ROCK, Rho kinase; $P L C$, phospholipase C; IP3, Inositol 1, 4, 5trisphosphate; TRPC1, transient receptor potential 1; NCX1, Na+/Ca 2+ exchanger 1; MaxiK, Ca2+-activated K+; PPARGC1, peroxisome proliferator-activated receptor-gamma coactivator-1; eNOS, endothelial nitric oxide synthase; VEGF-A, vascular endothelial growth factor $\mathrm{A}$; $P A I-1$, plasminogen activator inhibitor-1; $E M T$, epithelial-tomesenchymal transition; $C R P, C$-reactive protein; $C V D$, cardiovascular disease; VSMC, vascular smooth muscle cell (VSMC); ICAM1, intercellular adhesion molecule-1.

\section{Declarations}

\section{Ethics approval and consent to participate}

All the experiments were conducted in accordance with the National Institutes of Health Guide for the Care and Use of Laboratory Animals (NIH publication 85-23, revised 1996), and the experimental protocols were approved by the Institutional Animal Care and Use Committee (IACUC) of WuXi AppTec (Protocol Number: 27-2956). All surgery was performed under sodium pentobarbital anesthesia, and all efforts were made to minimize suffering.

\section{Consent for publication}

All authors agree to publication in the BMC Cardiovascular Disorders in English by Springer in Springer's corresponding English-language journal.

\section{Availability of data and material}

Microarray data have been deposited in NCBI's Gene Expression Omnibus under accession number GSE128167. (https://www.ncbi.nlm.nih.gov/geo/query/acc.cgi?acc = GSE128167)

\section{Competing interests}

All authors are the employee of Chengdu Kanghong Pharmaceutical Co., Ltd., Songling Xuemaikang Capsule is the product of our company. 


\section{Funding}

The present study was supported by the Secondary development of Chinese patent drug project (No. 2017SZ0035) and National Standardization of Traditional Chinese Medicine project (No. ZYBZH-C-SC-54).

\section{Authors' contributions}

All authors have contributed significantly and contributions as following: Li-tao LIU and Cui-1i Yan designed and performed experiments, Li-tao LIU and Qiao-xin Tang analyzed data and wrote the paper; Man-xi ZHAO and Chuanzhen TENG provided support in animal studies; Liang YE and Xiao KE designed the study, analyzed data, and reviewed the manuscript.

\section{Acknowledgements}

This work was supported by the Secondary development of Chinese patent drug (No. 2017SZ0035) and National Standardization of Traditional Chinese Medicine (No. ZYBZH-C-SC-54).

\section{Reference}

1. GBD 2013 Mortality and Causes of Death Collaborators. Global, regional, and national age-sex specific allcause and cause-specific mortality for 240 causes of death, 1990- 2013: A systematic analysis for the global burden of disease study 2013. Lancet. 2015; 385:117-171.

2. GBD Causes of Death Collaborators. Global, regional, and national age-sex specific mortality for 264 causes of death, 1980-2016: A systematic analysis for the global burden of disease study 2016. Lancet. 2017; 390:1151-1210.

3. Wu Y, Huxley R, Li L, Anna V, Xie G, Yao C, et al. Prevalence, awareness, treatment, and control of hypertension in china: Data from the china national nutrition and health survey 2002. Circulation. 2008; 118:2679-2686.

4. Wang Z, Chen Z, Zhang L, Wang X, Hao G, Zhang Z, et al. Status of Hypertension in China: Results From the China Hypertension Survey, 2012-2015. Circulation. 2018; 137:2344-2356.

5. Lloyd-Jones DM, Morris PB, Ballantyne CM, Birtcher KK, Daly DD Jr, DePalma SM, et al. 2017 Focused Update of the 2016 ACC Expert Consensus Decision Pathway on the Role of Non-Statin Therapies for LDL-Cholesterol Lowering in the Management of Atherosclerotic Cardiovascular Disease Risk: A Report of the American College of Cardiology Task Force on Expert Consensus Decision Pathways. J Am Coll Cardiol. 2017; 70:1785-1822.

6. WHO Library cataloguing in publication data. The regional strategy for traditional medicine in the western pacific(2011-2020). World Health organization 2012.

7. Sara S. Al Disi, M. Akhtar Anwar, Ali H. Eid. Anti-hypertensive herbs and their mechanisms of action:Part I. Front Pharmacol.2015; 6:323.

8. Wu XD, Wang C, Zhang ZY, Fu Y, Liu FY, Liu XH. Puerarin attenuates cerebral damage by improving cerebral microcirculation in spontaneously hypertensive rats.Evid Based Complement Alternat Med. 2014; 2014:408501

9. Li X, Lin Y, Zhou H, Li Y, Wang A, Wang H, et al. Puerarin protects against endothelial dysfunction and endorgan damage in Ang Il-induced hypertension.Clin Exp Hypertens. 2017; 39:58-64. 
10. Chen GH, Li YC, Lin NH, Kuo PC, Tzen JTC. Characterization of Vasorelaxant Principles from the Needles of Pinus morrisonicola Hayata. Molecules. 2017; 23. 86.

11. Liu LT, Liang L, Wang W, Yan CQ, Zhang J, Xiao YC, et al. Isolariciresinol-9'-O-a-L-arabinofuranoside protects against hydrogen peroxide-induced apoptosis of human umbilical vein endothelial cells via a PI3K/Akt/Bad-dependent pathway. Mol Med Rep. 2018; 17:488-494.

12. Shono M, Shimizu I, Aoyagi E, Taniguchi T, Takenaka H, Ishikawa M, et al. Reducing effect of feeding powdered nacre of Pinctada maxima on the visceral fat of rats. Biosci Biotechnol Biochem. 2008; 72:2761-2763.

13. Chen WQ, Chen FR. Effect of songling xuemaikang capsule combined with captopril on quality of life in primary hypertension patients. Zhongguo Zhong Xi Yi Jie He Za Zhi. 2001; 21:660-662.Chinese.

14. Fu L, Mao ZX, Wang J, Zhang J, Zheng TR, Wang SL. Effects of Songling Xuemaikang Capsule on ambulatory blood pressure in treatment of essential hypertension: a single-blind randomized controlled trial.Zhong Xi Yi Jie He Xue Bao. 2009; 7:509-513.Chinese.

15. Wong JL, Zheng Z. The clinical research of song ling xue mai kang ameliorating the compliance and endothelial function of artery in hypertensive patients. Journal of TCM Univ. of Hunan. 2011;31:6264.Chinese.

16. Ge CY. Effects of Songling Xuemaikang Capsule Combined with Valsartan on Essential Hypertension. Chinese Journal of integrative medicine on cardio-/cerebrovascular disease. 2012;10:515-516. Chinese.

17. Ritchie ME, Phipson B, Wu D, Hu YF, Law CW, Shi W, et al. Limma powers differential expression analyses for RNA-sequencing and microarray studies. Nucleic Acids Res. 2015; 43:e47.

18. Bolstad BM, Irizarry RA, Astrand M, Speed TP. A comparison of normalization methods for high density oligonucleotide array data based on variance and bias. Bioinformatics. 2003; 19:185-193.

19. Benjamini Y, Hochberg Y, Controlling the False Discovery Rate: a Practical and Powerful Approach to Multiple Testing. J R Stat Soc B. 1995; 57:289-300.

20. Nikolsky Y, Kirillov E, Zuev R, Rakhmatulin E, Nikolskaya T. Functional analysis of OMICs data and small molecule compounds in an integrated “knowledge-based” platform. Methods Mol Biol. 2009; 563:177-196.

21. Dezso Z, Nikolsky Y, Nikolskaya T, Miller J, Cherba D, Webb C, et al. Identifying disease-specific genes based on their topological significance in protein networks. BMC Syst Bio. 2009; 3: 36.

22. Vellaichamy A1, Dezso Z, JeBailey L, Chinnaiyan AM, Sreekumar A, Nesvizhskii Al, et al. "Topological significance" analysis of gene expression and proteomic profiles from prostate cancer cells reveals key mechanisms of androgen response. PLoS One. 2010; 5:e10936.

23. Chindelevitch L, Ziemek D, Enayetallah A, Randhawa R, Sidders B, Brockel C, et al. Causal reasoning on biological networks: interpreting transcriptional changes. Bioinformatics. 2012; 28:1114-1121.

24. Nikolsky Y, Sviridov E, Yao J, Dosymvekov D, Ustyansky V, Kaznacheev V, et al. Genome-wide functional synergy between amplified and mutated genes in human breast cancer. Cancer Res. 2008; 68: 9532-9540.

25. O'Leary NA, Wright MW, Brister JR, Ciufo S, Haddad D, Mcveigh R, et al. Reference sequence (RefSeq) database at NCBI: current status, taxonomic expansion,and functional annotation. Nucleic Acids Res. 2016; 44: D733745.

26. Kodera Y, Takeyama K, Murayama A, Suzawa M, Masuhiro Y, Kato S. Ligand typespecific interactions of peroxisome proliferator-activated receptory with transcriptional coactivators. J Biol Chem. 2000; 275:3320133204.

27. Lehrke M, Lazar MA. The many faces of PPARY. Cell. 2005; 123: 993-999.

Page $15 / 28$ 
28. Mettu NB, Stanley TB, Dwyer MA, Jansen MS, Allen JE, Hall JM, et al. The nuclear receptor-coactivator interaction surface as a target for peptide antagonists of the peroxisome proliferatoractivated receptors. Mol Endocrinol. 2007;21: 2361-2377.

29. Abe H, Semba H, Takeda N.The Roles of Hypoxia Signaling in the Pathogenesis of Cardiovascular Diseases.J Atheroscler Thromb. 2017; 24:884-894.

30. The UniProt, C., UniprotKB. 2017.

31. Kintscher U, Marx N, Martus P, Stoppelhaar M, Schimkus J, Schneider A, et al. Effect of high-dose valsartan on inflammatory and lipid parameters in patients with Type 2 diabetes and hypertension. Diabetes Res Clin Pract. 2010; 89:209-215.

32. Liu L, Zhao SP, Zhou HN, Li QZ, Li JX. Effect of fluvastatin and valsartan, alone and in combination, on postprandial vascular inflammation and fibrinolytic activity in patients with essential hypertension. J Cardiovasc Pharmacol. 2007; 50:50-55.

33. Otsuka T, Takada H, Nishiyama Y, Kodani E, Saiki Y, Kato K, et al. Dyslipidemia and the Risk of Developing Hypertension in a Working-Age Male Population. J Am Heart Assoc. 2016;5:e003053.

34. Coffman TM. Under pressure: the search for the essential mechanisms of hypertension. Nat Med. 2011;17:1402-1409.

35. Gaudio G, Guasti L, Schizzarotto A, Simoni C, Crespi C, Cimpanelli M, et al. Changes in plasma lipids during renin-angiotensin system blockade by combination therapy (enalapril plus valsartan) in patients with diabetes and hypertension. J Cardiovasc Pharmacol. 2005; 45:362-366.

36. Kintscher U, Marx N, Martus P, Stoppelhaar M, Schimkus J, Schneider A, et al. Effect of high-dose valsartan on inflammatory and lipid parameters in patients with Type 2 diabetes and hypertension. Diabetes Res Clin Pract. 2010; 89:209-215.

37. Hanefeld $\mathrm{M}$, Abletshauser $\mathrm{C}$. Effect of the angiotensin II receptor antagonist valsartan on lipid profile and glucose metabolism in patients with hypertension. J Int Med Res. 2001; 9:270-279.

38. Lee S, Kwak HB. Role of adiponectin in metabolic and cardiovascular disease. J Exerc Rehabil. 2014;10:54-59.

39. Goldstein BJ, Scalia RG, Ma XL. Protective vascular and myocardial effects of adiponectin. Nat Clin Pract Cardiovasc Med. 2009;6(1): 27-35.

40. Lopaciuk, S. [Inherited thrombophilia]. Acta Haematol Pol. 1994; 25: 41-54.Polish

41. Renna NF, de Las Heras N, Miatello RM. Pathophysiology of vascular remodeling in hypertension. Int J Hypertens. 2013; 2013:808353.

42. Intengan HD, Schiffrin EL. Vascular remodeling in hypertension: roles of apoptosis, inflammation, and fibrosis. Hypertension. 2001; 8:581-587.

43. Martinsen A, Dessy C, Morel N. Regulation of calcium channels in smooth muscle: new insights into the role of myosin light chain kinase. Channels (Austin). 2014; 8:402-413.

44. Jornayvaz FR, Shulman GI. Regulation of mitochondrial biogenesis. Essays Biochem. 2010; 47:69-84.

45. Eirin A, Lerman A, Lerman LO. Mitochondrial injury and dysfunction in hypertension-induced cardiac damage. Eur Heart J.2014; 35:3258-3266.

46. Zhang Y, Marsboom G, Toth PT, Rehman J. Mitochondrial respiration regulates adipogenic differentiation of human mesenchymal stem cells. PLoS One. 2013; 8: e77077. 
47. Castorena-Gonzalez JA, Staiculescu MC, Foote C, Martinez-Lemus LA. Mechanisms of the inward remodeling process in resistance vessels: is the actin cytoskeleton involved?. Microcirculation. 2014; 21:219-229.

48. Lemarié CA, Tharaux PL, Lehoux S. Extracellular matrix alterations in hypertensive vascular remodeling. J Mol Cell Cardiol. 2010; 48:433-439.

49. Fogari R, Zoppi A. Antihypertensive drugs and fibrinolytic function. Am J Hypertens. 2006; 19:1293-1299.

50. Kovacic JC, Mercader N, Torres M, Boehm M, Fuster V. Epithelial-to-mesenchymal and endothelial-tomesenchymal transition: from cardiovascular development to disease. Circulation. 2012; 125:1795-1808.

51. Markiewski MM, Lambris JD. The role of complement in inflammatory diseases from behind the scenes into the spotlight. Am J Pathol. 2007; 171:715-727.

52. Wenzel UO, Bode M, Kőhl J, Ehmke H. A pathogenic role of complement in arterial hypertension and hypertensive end organ damage. Am J Physiol Heart Circ Physiol. 2017; 312:H349-H354.

53. Carter AM. Complement activation: an emerging player in the pathogenesis of cardiovascular disease. Scientifica (Cairo). 2012; 2012: 402783.

54. Savoia C, Sada L, Zezza L, Pucci L, Lauri FM, Befani A, et al. Vascular inflammation and endothelial dysfunction in experimental hypertension. Int J Hypertens. 2011; 2011:281240.

\section{Tables}

Table 1. The systolic blood pressure (SBP) diastolic blood pressure (DBP) of the rats in different groups (mean \pm SE, $\mathrm{mmHg}$ )

\begin{tabular}{|c|c|c|c|c|c|c|c|}
\hline & \multirow[t]{2}{*}{ Groups } & \multirow{2}{*}{$\begin{array}{l}\text { Animal } \\
\text { number }\end{array}$} & \multicolumn{5}{|c|}{ Weeks } \\
\hline & & & 0 & 1 & 2 & 3 & 4 \\
\hline \multirow[t]{4}{*}{ SBP } & Control group & 8 & $186.04 \pm 4.19$ & $198.83 \pm 3.57$ & $207.58 \pm 4.85$ & $205.08 \pm 4.89$ & $210.38 \pm 2.40$ \\
\hline & $\begin{array}{l}\text { Valsartan } \\
\text { group }\end{array}$ & 6 & $193.43 \pm 2.23$ & $170.21 \pm 2.68^{* * *}$ & $164.00 \pm 2.96^{* * *}$ & $168.40 \pm 2.89^{* * *}$ & $168.39 \pm 3.89^{* * *}$ \\
\hline & $\begin{array}{l}\text { SXC low-dose } \\
\text { group }\end{array}$ & 6 & $186.46 \pm 4.97$ & $186.33 \pm 5.04$ & $182.68 \pm 4.01^{* *}$ & $183.72 \pm 3.50^{* *}$ & $181.98 \pm 3.77^{* * *}$ \\
\hline & $\begin{array}{l}\text { SXC high-dose } \\
\text { group }\end{array}$ & 6 & $193.24 \pm 3.13$ & $190.20 \pm 4.75$ & $190.95 \pm 7.06$ & $185.85 \pm 1.45^{* *}$ & $196.04 \pm 5.38^{*}$ \\
\hline \multirow[t]{4}{*}{ DBP } & Control group & 8 & $138.88 \pm 5.13$ & $156.67 \pm 4.01$ & $156.79 \pm 8.51$ & $164.63 \pm 4.54$ & $172.58 \pm 2.60$ \\
\hline & $\begin{array}{l}\text { Valsartan } \\
\text { group }\end{array}$ & 6 & $134.31 \pm 3.81$ & $111.02 \pm 2.62^{* * *}$ & $107.46 \pm 1.67^{* * *}$ & $103.27 \pm 1.63^{* * *}$ & $113.46 \pm 3.79^{* * *}$ \\
\hline & $\begin{array}{l}\text { SXC low-dose } \\
\text { group }\end{array}$ & 6 & $126.70 \pm 5.23$ & $122.58 \pm 6.43^{* * *}$ & $121.51 \pm 4.71^{* * *}$ & $123.32 \pm 4.45^{* * *}$ & $121.54 \pm 3.73^{* * *}$ \\
\hline & $\begin{array}{l}\text { SXC high-dose } \\
\text { group }\end{array}$ & 6 & $130.39 \pm 1.94$ & $127.24 \pm 4.43^{* * *}$ & $121.86 \pm 4.52^{* * *}$ & $122.88 \pm 0.82^{* * *}$ & $126.30 \pm 2.72^{* * *}$ \\
\hline
\end{tabular}

$P$ values werer calculated using the Two-way ANOVA, $* P<0.05 \rrbracket^{* *} P<0.01 \rrbracket^{* * *} P<0.001$ vs. control group.

Table 2. Topologically significant genes (TSGs ) for each sample group, ranked by adjusted P value. 


\begin{tabular}{|c|c|c|c|c|c|c|c|}
\hline \multirow[t]{2}{*}{ TSGs Category } & \multirow[t]{2}{*}{ Rank } & \multirow{2}{*}{$\begin{array}{c}\text { Valsartan } \\
\text { Name }\end{array}$} & \multicolumn{3}{|c|}{ SXC low-dose } & \multicolumn{2}{|l|}{ SXC high-dose } \\
\hline & & & $\begin{array}{l}\text { Adjusted } \\
\mathrm{P} \text { value }\end{array}$ & Name & $\begin{array}{l}\text { Adjusted } \\
\mathrm{P} \text { value }\end{array}$ & Name & $\begin{array}{r}\text { Adjusted } \\
\text { value }\end{array}$ \\
\hline \multirow[t]{5}{*}{ Overconnected } & 1 & SP1 & $1.23 \mathrm{E}-12$ & SP1 & $7.37 \mathrm{E}-13$ & SP1 & $2.37 \mathrm{E}-05$ \\
\hline & 2 & $\begin{array}{l}\text { PPAR-alpha/RXR- } \\
\text { alpha }\end{array}$ & $6.93 \mathrm{E}-11$ & GCR & $9.21 \mathrm{E}-09$ & HIF1A & 4.25E-05 \\
\hline & 3 & $\begin{array}{l}\text { PPAR- } \\
\text { gamma/RXR- } \\
\text { alpha }\end{array}$ & $1.34 \mathrm{E}-07$ & $\begin{array}{l}\text { PPAR- } \\
\text { alpha/RXR- } \\
\text { alpha }\end{array}$ & $1.64 \mathrm{E}-07$ & HNF3-beta & $6.56 \mathrm{E}-05$ \\
\hline & 4 & PPAR-gamma & 2.71E-07 & GATA-2 & $4.24 \mathrm{E}-07$ & HIF-1 & 9.44E-05 \\
\hline & 5 & ERR3 & 1.83E-06 & $\begin{array}{l}\text { PPAR- } \\
\text { gamma/RXR- } \\
\text { alpha }\end{array}$ & $1.31 \mathrm{E}-06$ & $\begin{array}{l}\text { PPAR- } \\
\text { gamma/RXR- } \\
\text { alpha }\end{array}$ & 0.000130829 \\
\hline \multirow[t]{5}{*}{ Hidden nodes } & 1 & PPAR-gamma & 3.72E-12 & PLAUR(UPAR) & $9.81 \mathrm{E}-15$ & PLAUR(uPAR) & 7.27E-15 \\
\hline & 2 & MEF2D & $3.15 \mathrm{E}-10$ & c-Fos & $1.60 \mathrm{E}-12$ & ADAM12 & 1.63E-13 \\
\hline & 3 & LXR-alpha & $3.66 \mathrm{E}-10$ & Fibronectin & $1.98 \mathrm{E}-12$ & Fibronectin & $4.48 \mathrm{E}-13$ \\
\hline & 4 & SP1 & $5.32 \mathrm{E}-10$ & ADAM12 & $1.78 \mathrm{E}-11$ & c-Fos & $1.06 \mathrm{E}-10$ \\
\hline & 5 & NF_AT1(NFATC2) & 3.75E-09 & L-selectin & $1.81 \mathrm{E}-11$ & MMP-13 & $3.38 \mathrm{E}-10$ \\
\hline \multirow{5}{*}{$\begin{array}{l}\text { Causal } \\
\text { reasoning } \\
\text { hyotheses }\end{array}$} & 1 & MEK1(MAP2K1) & $1.91 \mathrm{E}-08$ & $\begin{array}{l}\text { Thrombospondin } \\
2\end{array}$ & 5.77E-07 & ZNF706 & 2.97E-07 \\
\hline & 2 & CNOT3 & $1.23 \mathrm{E}-07$ & PRK1 & 3.09E-06 & $\begin{array}{l}\text { SP1/SP3 } \\
\text { complex }\end{array}$ & $3.18 \mathrm{E}-07$ \\
\hline & 3 & $\begin{array}{l}\text { PRC(PGC-1 } \\
\text { related) }\end{array}$ & 1.87E-07 & SSPH1 & 3.87E-06 & TLE2 & 4.43E-07 \\
\hline & 4 & HES1 & 2.67E-07 & CNOT3 & 4.01E-06 & ARR19 & 7.07E-07 \\
\hline & 5 & DSIPI(GILZ) & $3.52 \mathrm{E}-07$ & Dynamin-3 & 4.02E-06 & CNOT3 & 7.07E-07 \\
\hline
\end{tabular}

Table 3. Top 10 key pathway maps for SXC high-dose treatment

\begin{tabular}{lccc}
\hline \multicolumn{1}{c}{ Key Mpas ID } & Genelist & TSgenes & Union \\
\hline $\begin{array}{l}\text { Putative pathways of activation of classical complement system in major depressive } \\
\text { disorder }\end{array}$ & $4.02 \mathrm{E}-05$ & 0.000965477 & $9.91 \mathrm{E}-06$ \\
Role of iNKT and B cells in T cell recruitment in allergic contact dermatitis & 0.000567424 & $2.02 \mathrm{E}-08$ & $8.19 \mathrm{E}-09$ \\
\hline Development_Role of G-CSF in hematopoietic stem cell mobilization & 0.0007310930 .000293084 & $7.88 \mathrm{E}-07$ \\
\hline Role of cell adhesion in vaso-occlusion in Sickle cell disease & 0.00077053 & 0.000903643 & $2.11 \mathrm{E}-06$ \\
\hline Regulation of lipid metabolism_G-alpha(q) regulation of lipid metabolism & 0.0009716280 .0066373350 .000118804 \\
\hline Immune response_Regulatory role of C1q in platelet activation & 0.001226119 & $7.51 \mathrm{E}-05$ & $1.20 \mathrm{E}-05$ \\
\hline Role of adipose tissue hypoxia in obesity and type 2 diabetes & 0.002240057 & $2.03 \mathrm{E}-05$ & $2.26 \mathrm{E}-08$ \\
\hline $\begin{array}{l}\text { Vascular endothelial cell damage in SLE } \\
\text { Pioglitazone and Rosiglitazone in treatment of type 2 diabetes and metabolic }\end{array}$ & 0.0025199130 .0168298920 .000405042 \\
\hline syndrome X & 0.002665178 & $1.92 \mathrm{E}-05$ & $2.12 \mathrm{E}-06$ \\
\hline Neutrophil adhesion and transendothelial migration in asthma & 0.0032731910 .0278034230 .000506431 \\
\hline
\end{tabular}

Genelist, P value fro enrichment with DEGs; Tsgenes, P value for enrichment with topologically significant genes; Union, $\mathrm{P}$ value for enrichment with DEGs \& Tsgens

Table 4. Top 10 key pathway maps for valsartan treatment 


\begin{tabular}{|c|c|c|c|}
\hline Key Mpas ID & Genelist & TSgenes & Union \\
\hline $\begin{array}{l}\text { Pioglitazone and Rosiglitazone in treatment of type } 2 \text { diabetes and metabolic } \\
\text { syndrome X }\end{array}$ & $9.70 \mathrm{E}-06$ & \multicolumn{2}{|c|}{$0.0017434854 .43 \mathrm{E}-06$} \\
\hline Role of cell adhesion in vaso-occlusion in Sickle cell disease & \multicolumn{3}{|c|}{$0.0003076960 .0053493288 .79 \mathrm{E}-05$} \\
\hline Development_Beta adrenergic receptors in brown adipocyte differentiation & \multicolumn{2}{|c|}{$0.0007950329 .48 \mathrm{E}-06$} & $1.30 \mathrm{E}-07$ \\
\hline Lipoprotein metabolism & \multicolumn{3}{|c|}{$0.0009148450 .0004468351 .60 \mathrm{E}-05$} \\
\hline VLDL, LDL dyslipidemia in type 2 diabetes and metabolic syndrome $\mathrm{X}$ & \multicolumn{3}{|c|}{0.0012367130 .0234759420 .000203458} \\
\hline Regulation of lipid metabolism_G-alpha(q) regulation of lipid metabolism & \multicolumn{3}{|c|}{$0.0013955590 .0005324556 .12 \mathrm{E}-05$} \\
\hline Development_Transcriptional regulation of megakaryopoiesis & \multicolumn{3}{|c|}{0.0014027340 .0124586010 .000400422} \\
\hline Transport_HDL-mediated reverse cholesterol transport & \multicolumn{3}{|c|}{0.0026251040 .0014442610 .00018817} \\
\hline Role of adipose tissue hypoxia in obesity and type 2 diabetes & \multicolumn{3}{|c|}{0.0029944690 .0280615060 .000497865} \\
\hline Regulation of IGF family activity in colorectal cancer & \multicolumn{3}{|c|}{0.0034459840 .0096850750 .000195788} \\
\hline
\end{tabular}

Genelist, P value fro enrichment with DEGs; Tsgenes, P value for enrichment with topologically significant genes; Union, $\mathrm{P}$ value for enrichment with DEGs \& Tsgens

Table 5 Overlapping pathways between SXC and valsartan treatment.

$\begin{array}{lll}\text { Key pathway ID } & \text { PCD } & \text { SXC high-dose }\end{array}$

Genelist Tsgenes Union Genelist Tsgenes Union

Regulation of lipid metabolism_G-alpha(q) $\quad 0.0013955590 .0005324556 .12 \mathrm{E}-05 \quad 0.0009716280 .0066373350 .000118804$
regulation of lipid metabolism

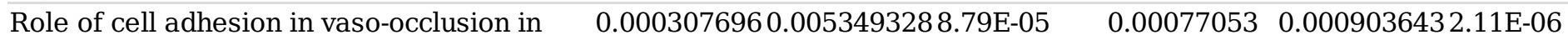
Sickle cell disease

Role of iNKT and B cells in T cell recruitment in allergic contact dermatitis
$0.0100123480 .0138360170 .0006120860 .0005674242 .02 \mathrm{E}-08$
8.19E-09 Lipoprotein metabolism

Development_Transcriptional regulation of megakaryopoiesis

Aminoglycoside- and cisplatin-induced hair cell death

Stem cells_TGF-beta family mediated differentiation of embryonic stem cells

$0.0009148450 .0004468351 .60 \mathrm{E}-05 \quad 0.0047864083 .09 \mathrm{E}-05 \quad 8.38 \mathrm{E}-07$

0.0014027340 .0124586010 .0004004220 .0046350580 .0091036360 .000889546

0.031702221 8.18E-05 1.55E-05 0.042496747 0.000307371 2.52E-05

$0.0458179930 .02602198 \quad 0.0019499710 .0370934720 .0002211236 .58 \mathrm{E}-05$

0.0012367130 .0234759420 .0002034580 .0307671910 .0004240760 .000118927 VLDL, LDL dyslipidemia in type 2 diabetes and metabolic syndrome $\mathrm{X}$

Immune response_IL-10 signaling pathway 0.0059645820 .0485424390 .0012867470 .0307334420 .0347271250 .001595638 Pioglitazone and Rosiglitazone in treatment 9.70E-06 $\quad 0.0017434854 .43 \mathrm{E}-06 \quad 0.0026651781 .92 \mathrm{E}-05 \quad 2.12 \mathrm{E}-06$ of type 2 diabetes and metabolic syndrome $\mathrm{X}$

Role of Diethylhexyl Phthalate and

Tributyltin in fat cell differentiation

Vascular endothelial cell damage in SLE

Role of adipose tissue hypoxia in obesity and type 2 diabetes

Development_Differentiation of white adipocytes

Transport_HDL-mediated reverse cholesterol transport

Main genetic and epigenetic alterations in lung cancer

Genelist, P value fro enrichment with DEGs; Tsgenes, P value for enrichment with topologically significant genes; Union, $\mathrm{P}$
$0.0059512664 .78 \mathrm{E}-05$
1.60E-05
$0.02582707 \quad 0.0003104937 .24 \mathrm{E}-05$

0.0251609220 .0003611760 .0001415410 .0025199130 .0168298920 .000405042 $0.0029944690 .0280615060 .0004978650 .0022400572 .03 \mathrm{E}-05 \quad 2.26 \mathrm{E}-08$
0.0079277453.88E-09
2.32E-09
$0.0178711090 .0001099051 .19 \mathrm{E}-05$

$\begin{array}{lllll}0.0026251040 .0014442610 .00018817 & 0.0140205410 .00106627 & 0.000115123\end{array}$

0.0398177370 .0063270180 .0013589190 .0102761750 .0150249710 .002217789 value for enrichment with DEGs \& Tsgens 
Table 6. Key pathway maps unique to SXC high-dose treatment group.

\begin{tabular}{|c|c|c|c|}
\hline Key pathway ID & Genelist & Tsgenes & Union \\
\hline $\begin{array}{l}\text { Putative pathways of activation of classical complement system in major } \\
\text { depressive disorder }\end{array}$ & $4.02 \mathrm{E}-05$ & \multicolumn{2}{|c|}{$0.0009654779 .91 \mathrm{E}-06$} \\
\hline Development_Role of G-CSF in hematopoietic stem cell mobilization & \multicolumn{3}{|c|}{$0.0007310930 .0002930847 .88 \mathrm{E}-07$} \\
\hline Immune response_Regulatory role of C1q in platelet activation & \multicolumn{3}{|c|}{$0.0012261197 .51 \mathrm{E}-05 \quad 1.20 \mathrm{E}-05$} \\
\hline Neutrophil adhesion and transendothelial migration in asthma & \multicolumn{3}{|c|}{0.0032731910 .0278034230 .000506431} \\
\hline Transcription_HIF-1 targets & \multicolumn{3}{|c|}{$0.0049652793 .05 \mathrm{E}-05 \quad 1.49 \mathrm{E}-07$} \\
\hline IGF signaling in lung cancer & \multicolumn{3}{|c|}{$0.0072570250 .0006821977 .95 \mathrm{E}-05$} \\
\hline Eosinophil adhesion and transendothelial migration in asthma & \multicolumn{2}{|c|}{$0.0099570341 .38 \mathrm{E}-07$} & 1.19E-08 \\
\hline Immune response_Role of integrins in NK cells cytotoxicity & \multicolumn{3}{|c|}{$0.0128649790 .00026858 \quad 8.89 \mathrm{E}-05$} \\
\hline Immune response_IL-5 signaling via PI3K, MAPK and NF-kB & \multicolumn{2}{|c|}{$0.0140855863 .00 \mathrm{E}-07$} & $9.14 \mathrm{E}-09$ \\
\hline Stem cells_NOTCH1-induced self-renewal of glioblastoma stem cells & \multicolumn{2}{|c|}{$0.0145821899 .35 \mathrm{E}-06$} & $1.05 \mathrm{E}-06$ \\
\hline Development_Astrocyte differentiation from adult stem cells & \multicolumn{3}{|c|}{0.0145821890 .0066373350 .00047126} \\
\hline Th17 cells in CF & \multicolumn{3}{|c|}{0.0167744360 .0066197670 .001358136} \\
\hline Autocrine production of eosinophil pro-surviv & \multicolumn{3}{|c|}{0.0184960710 .0021337790 .000205287} \\
\hline Cell adhesion_PLAU signaling & \multicolumn{3}{|c|}{$0.0207063 \quad 0.00246708 \quad 0.00026588$} \\
\hline $\begin{array}{l}\text { Inflammatory factors-induced expression of mucins in normal and asthmatic } \\
\text { epithelium }\end{array}$ & 0.0207063 & \multicolumn{2}{|c|}{0.0096328640 .000961138} \\
\hline Retinoic acid regulation of oligodendrocyte differentiation in multiple sclerosis & \multicolumn{3}{|c|}{$0.02582707 \quad 0.0156692440 .000500631$} \\
\hline Epithelial cell apoptosis in COPD & \multicolumn{3}{|c|}{0.0261232960 .0106989430 .001143086} \\
\hline Role of CD8+ Tc1 cells in COPD & \multicolumn{3}{|c|}{$0.0263452528 .62 \mathrm{E}-06 \quad 2.11 \mathrm{E}-06$} \\
\hline IL-6 signaling in tumor-associated monocytes/macrophages in breast cancer & \multicolumn{3}{|c|}{$0.0331934120 .0002303838 .89 \mathrm{E}-05$} \\
\hline Immune response_Role of HMGB1 in dendritic cell maturation and migration & \multicolumn{3}{|c|}{0.0333185450 .0172725230 .000836226} \\
\hline Role of B cells in SLE & \multicolumn{3}{|c|}{0.0365341830 .0364254670 .004832008} \\
\hline Development_Leptin signaling via JAK/STAT and MAPK cascades & \multicolumn{3}{|c|}{$0.0383059920 .0049297 \quad 0.00027204$} \\
\hline Hypertrophy of asthmatic airway smooth muscle cells & \multicolumn{3}{|c|}{0.0386670570 .0001252980 .000110628} \\
\hline Breast cancer (general schema) & \multicolumn{3}{|c|}{0.0411315540 .0216234030 .004471035} \\
\hline Mechanisms of CAM-DR in multiple myeloma & \multicolumn{3}{|c|}{0.0414554750 .0060822960 .003861679} \\
\hline Neuroendocrine transdifferentiation in Prostate Cancer & \multicolumn{3}{|c|}{0.0414554750 .020278220 .010563399} \\
\hline Proinflammatory cytokine production by Th17 cells in asthma & \multicolumn{3}{|c|}{0.0472398370 .0021864610 .000555421} \\
\hline Role of IL- 6 in obesity and type 2 diabetes in adipocytes & \multicolumn{3}{|c|}{$0.0484628944 .54 \mathrm{E}-06 \quad 3.54 \mathrm{E}-06$} \\
\hline IL-6 signaling in breast cancer cells & \multicolumn{3}{|c|}{0.0492214580 .0076267130 .001894012} \\
\hline $\begin{array}{l}\text { Transcription_Role of the non-genomic action of Retinoic acid and } \\
\text { phosphorylation of Retinoic acid receptors in the initiation of transcripti }\end{array}$ & \multicolumn{3}{|c|}{0.0492214580 .0244197} \\
\hline
\end{tabular}

Genelist, $\mathrm{P}$ value fro enrichment with DEGs; Tsgenes, $\mathrm{P}$ value for enrichment with topologically significant genes; Union, $\mathrm{P}$ value for enrichment with DEGs \& Tsgens

Table 7. Key pathway maps unique to valsartan treatment group. 


\begin{tabular}{|c|c|c|c|}
\hline Key pathway ID & Genelist & Tsgenes & Union \\
\hline Development_Beta adrenergic receptors in brown adipocyte differentiation & 0.000795032 & $9.48 \mathrm{E}-06$ & $1.30 \mathrm{E}-07$ \\
\hline Regulation of IGF family activity in colorectal cancer & \multicolumn{3}{|c|}{0.0034459840 .0096850750 .000195788} \\
\hline Adiponectin in pathogenesis of type 2 diabetes & \multicolumn{3}{|c|}{$0.0042922020 .011559631 \quad 5.09 \mathrm{E}-05$} \\
\hline Role of ZNF202 in regulation of expression of genes involved in atherosclerosis & \multicolumn{3}{|c|}{0.0042922020 .000339496} \\
\hline $\begin{array}{l}\text { Aberrant lipid trafficking and metabolism in age-related macular degeneration } \\
\text { pathogenesis }\end{array}$ & \multicolumn{3}{|c|}{$0.004763555 \quad 1.85 \mathrm{E}-05 \quad 9.43 \mathrm{E}-06$} \\
\hline Immune response_HSP60 and HSP70/ TLR signaling pathway & 0.005254697 & 4.01E-05 & 2.93E-06 \\
\hline $\begin{array}{l}\text { Oxidative stress in adipocyte dysfunction in type } 2 \text { diabetes and metabolic } \\
\text { syndrome X }\end{array}$ & \multicolumn{3}{|c|}{0.0081647720 .0286729860 .001013013} \\
\hline Development_Neural stem cell lineage commitment (schema) & \multicolumn{3}{|c|}{0.0083939350 .0162369210 .000174023} \\
\hline Protein folding and maturation_Angiotensin system maturation $\backslash$ Rodent & \multicolumn{3}{|c|}{0.0092519560 .0214936770 .004577634} \\
\hline Stem cells_BMP signaling in cardiac myogenesis & \multicolumn{3}{|c|}{0.0097342180 .0047306020 .000236524} \\
\hline NETosis in SLE & \multicolumn{3}{|c|}{0.0113142340 .0274309350 .002853749} \\
\hline Development_Oligodendr & 0.015393263 & $4.91 \mathrm{E}-07$ & $4.28 \mathrm{E}-07$ \\
\hline Maturation and migration of dendritic cells in skin sensitization & \multicolumn{3}{|c|}{0.0153932630 .0019268670 .000149353} \\
\hline $\begin{array}{l}\text { Development_Retinoic acid and retinoic acid receptors in regulation of } \\
\text { oligodendrocyte differentiation }\end{array}$ & \multicolumn{3}{|c|}{$0.0166770230 .001743485 \quad 3.19 \mathrm{E}-05$} \\
\hline Fenofibrate in treatment of type 2 diabetes and metabolic syndrome $\mathrm{X}$ & \multicolumn{3}{|c|}{0.0166770230 .0002567210 .000195788} \\
\hline CREB1-dependent transcription deregulation in Huntington's Disease & \multicolumn{3}{|c|}{0.0197732280 .0115596310 .00143771} \\
\hline Protein folding and maturation_Angiotensin system maturation $\backslash$ Human version & \multicolumn{3}{|c|}{0.0197732280 .0115596310 .006033284} \\
\hline $\begin{array}{l}\text { TNF-alpha, IL-1 beta induce dyslipidemia and inflammation in obesity and type } 2 \\
\text { diabetes in adipocytes }\end{array}$ & \multicolumn{3}{|c|}{$0.0212213490 .000636714 \quad 8.30 \mathrm{E}-05$} \\
\hline Transport_Low density lipoproteins assembly and remodeling & \multicolumn{3}{|c|}{0.0227267670 .0158560440 .003336313} \\
\hline LRRK2 and immune function in Parkinson's disease & \multicolumn{3}{|c|}{0.0259587590 .0034476550 .000537509} \\
\hline Effect of H. pylori infection on apoptosis in gastric epithelial cells & \multicolumn{3}{|c|}{0.0259797770 .0037423480 .001520347} \\
\hline Th1 cells in dry eye & \multicolumn{3}{|c|}{$0.0294694320 .000355774 \quad 6.46 \mathrm{E}-05$} \\
\hline $\begin{array}{l}\text { Regulation of lipid metabolism_Regulation of fatty acid synthesis: NLTP and } \\
\text { EHHADH }\end{array}$ & \multicolumn{3}{|c|}{0.0297833050 .0023905290 .000702703} \\
\hline Development_Beta-adrenergic receptors signaling via Cyclic AMP & \multicolumn{3}{|c|}{0.0313689350 .0047635780 .002224803} \\
\hline iNKT cell-keratinocyte interactions in allergic contact dermatitis & \multicolumn{3}{|c|}{$0.0370040560 .001245507 \quad 7.50 \mathrm{E}-05$} \\
\hline Reactive oxygen and nitrogen species production in eosinophils in asthma & \multicolumn{3}{|c|}{0.0370040560 .0207400630 .004091714} \\
\hline Macrophage and dendritic cell phenotype shift in cancer & \multicolumn{3}{|c|}{0.0397418390 .0024900690 .00093147} \\
\hline Niacin-HDL metabolism & \multicolumn{3}{|c|}{0.0425429840 .0053286390 .001738605} \\
\hline Regulation of metabolism_Role of Adiponectin in regulation of metabolism & \multicolumn{3}{|c|}{0.0458179930 .0072890750 .000550557} \\
\hline $\begin{array}{l}\text { SHH signaling in oligodendrocyte precursor cells differentiation in multiple } \\
\text { sclerosis }\end{array}$ & \multicolumn{3}{|c|}{0.0469193170 .0333309460 .002120527} \\
\hline Stem cells_NODAL signaling in early mesendoderm formation & \multicolumn{3}{|c|}{0.049444110 .0065830150 .000484669} \\
\hline
\end{tabular}

Genelist, P value fro enrichment with DEGs; Tsgenes, P value for enrichment with topologically significant genes; Union, P value for enrichment with DEGs \& Tsgens

\section{Figures}


A

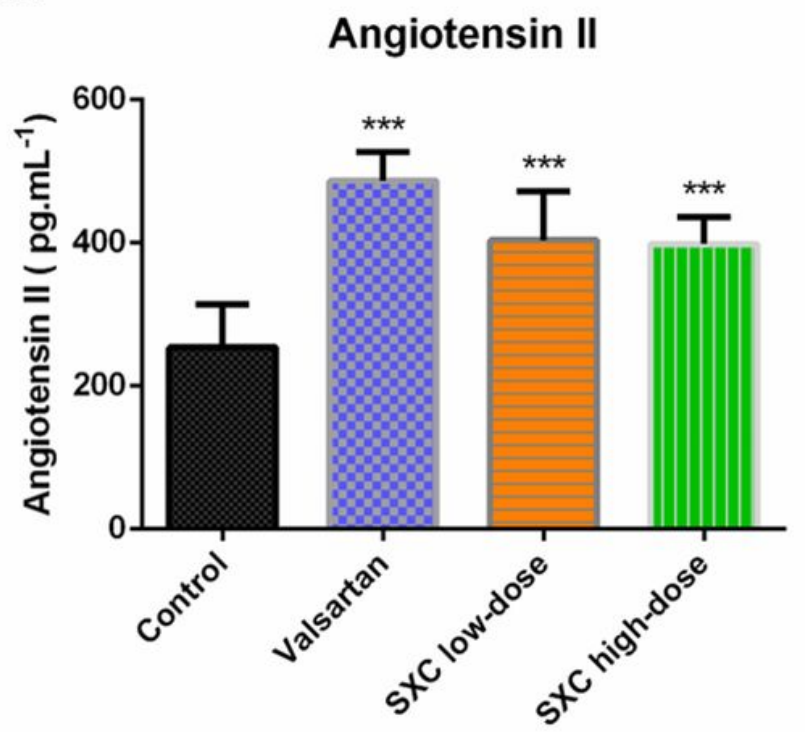

B

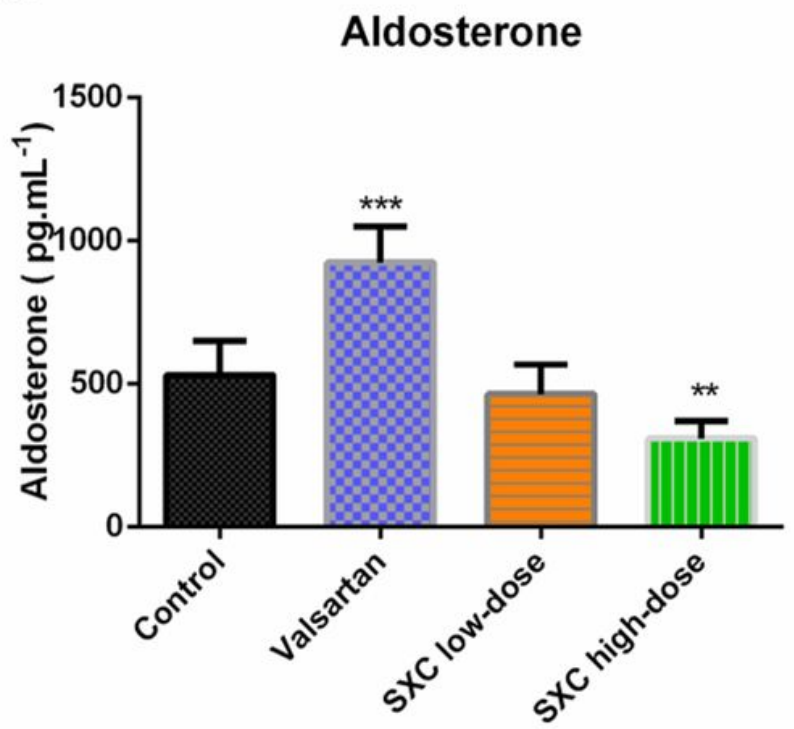

\section{Figure 1}

The content of angiotensin II and aldosterone in rats serum. (A) The serum angiotensin II levels in different groups; (B) The serum aldosterone levels in different groups. Control group $(n=8)$, the model group rats were orally administered with saline $(7.5 \mathrm{~mL} \cdot \mathrm{kg}-1)$ for 4 weeks; valsartan group $(\mathrm{n}=6)$, rats were orally administered with valsartan $(21.6 \mathrm{mg} \cdot \mathrm{kg}-1)$ for 4 weeks; SXC low-dose group $(n=6)$, rats were orally administered with SXC (236.25 $\mathrm{mg} \cdot \mathrm{kg}$-1) for 4 weeks; SXC high-dose group $(\mathrm{n}=6)$, rats were orally administered with SXC $(708.75 \mathrm{mg} \cdot \mathrm{kg}-1)$ for 4 weeks. (Compared with control group, $* * \mathrm{P}<0.01$, *** $\mathrm{P}<0.001$ ).

A
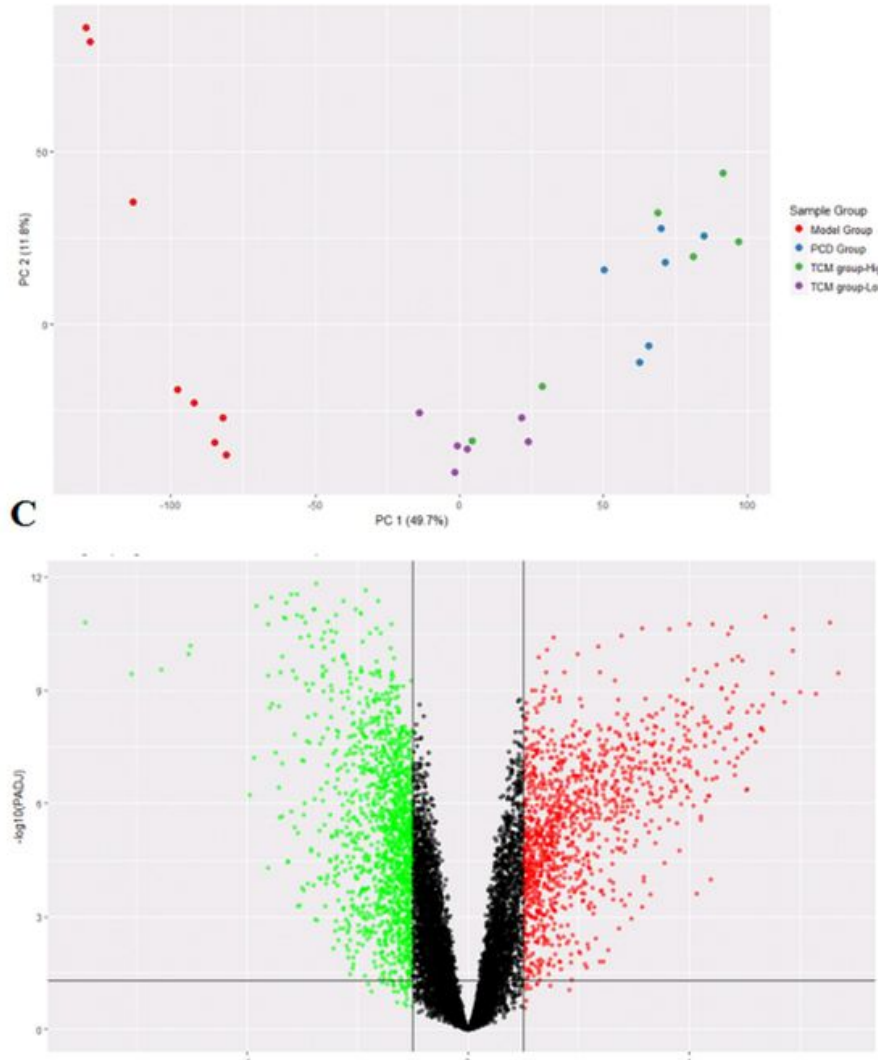

B
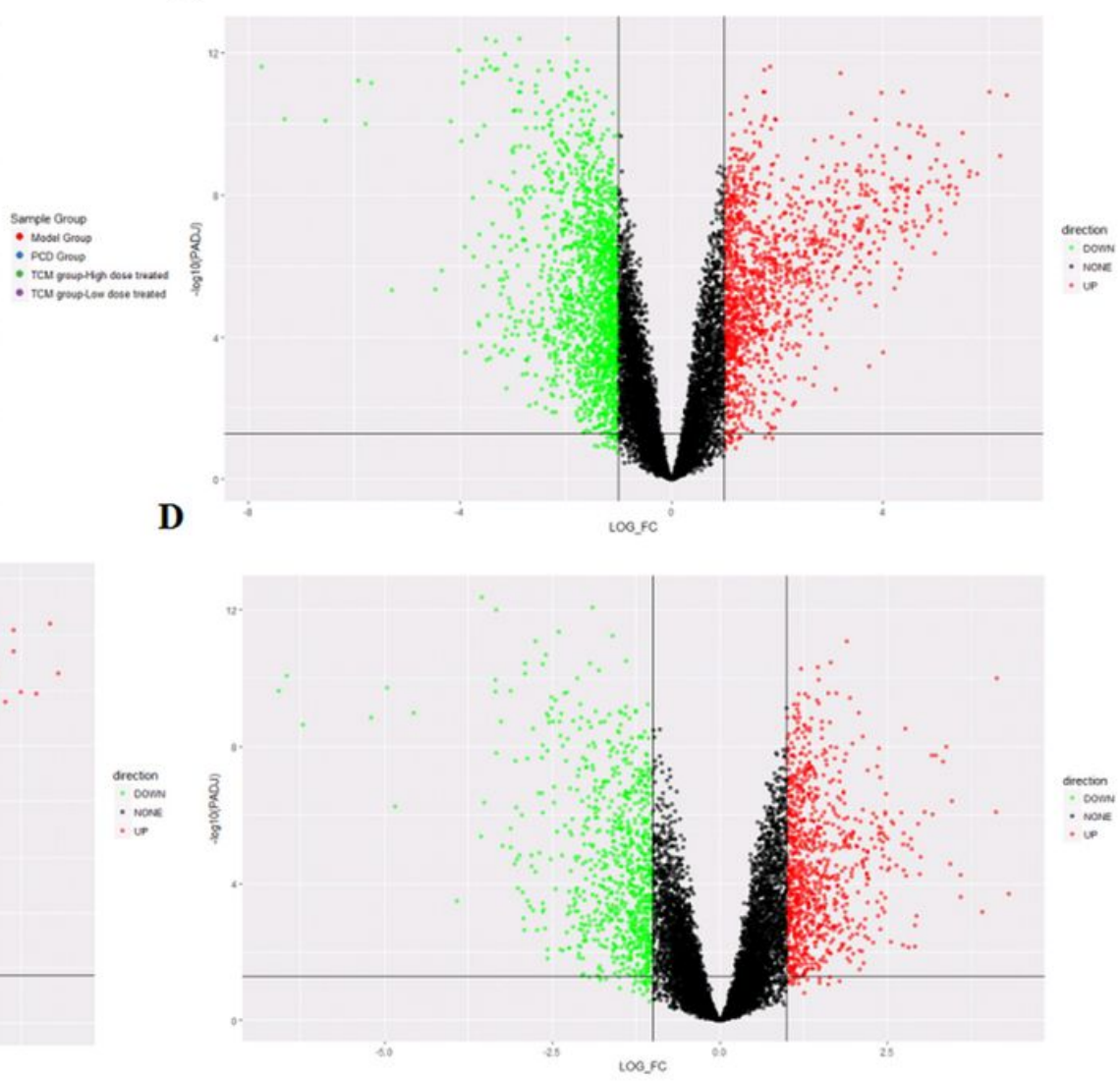
Figure 2

Identification and hierarchical clustering of DEGs. (A).Principal components analysis plot of samples,colored by sample group (PCD group: valsartan treated group). Volcano plot of DEGs in valsartan group compared to model group (B), SXC low-dose group compared to model group (C) and SXC high-dose group compared to model group (D). The cut-off criteria were $|\log 2 \mathrm{FC}|>1$ and $\mathrm{P}<0.05$. The red dots represented the up-regulated genes, and the green dots denoted the down-regulated genes. The gray dots indicated the genes with a $|\log 2 \mathrm{FC}|<1$ and/or $P>0.05$. (E) Heat map of the top $20 \%$ DEGs. Horizontal band with the cluster tree at the top: green, control group samples; red, treated group samples. Each row represented a single gene: green, downregulated DEGs; red, upregulated DEGs. The depth of the color denoted the change degree.

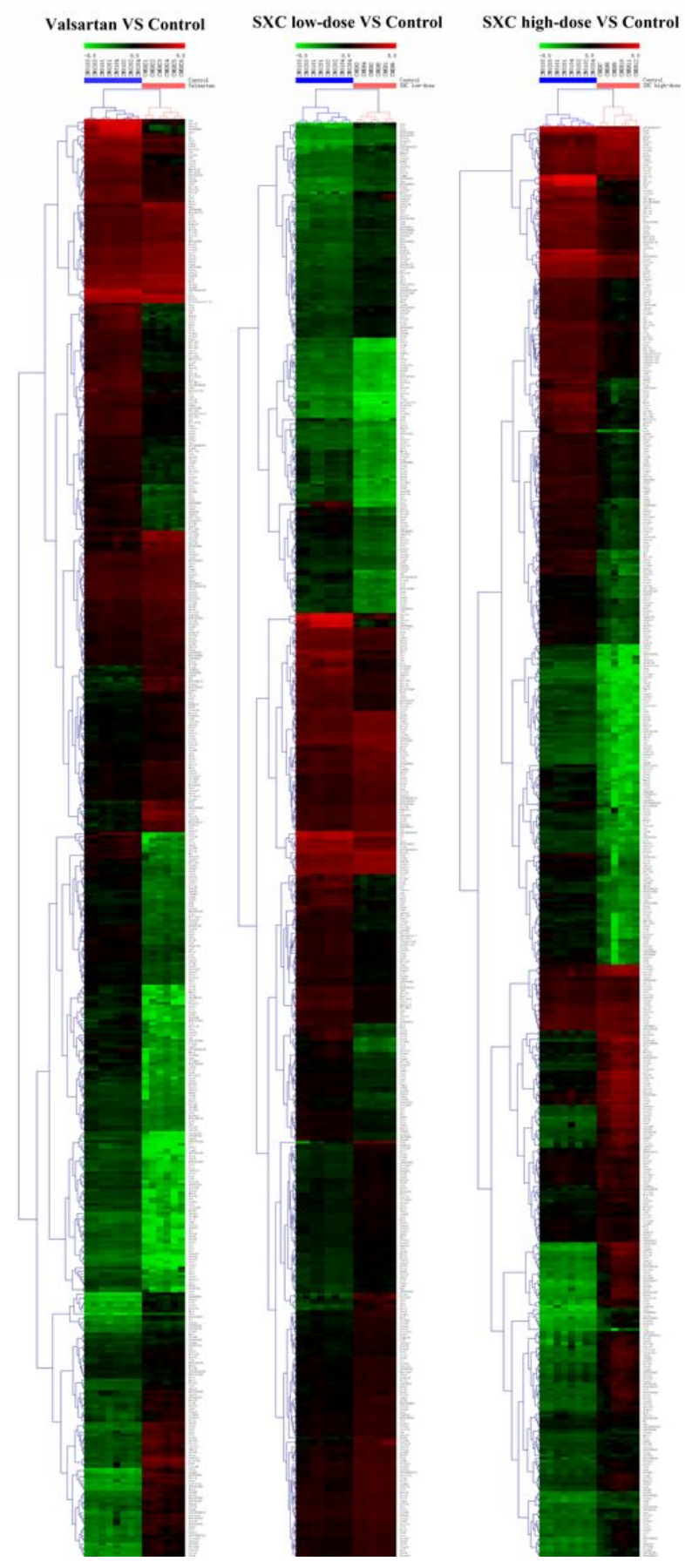

Figure 3 
Heat map of the top 500 DEGs in different treatment groups compared to the control group. Horizontal band with the cluster tree at the top: Blue, control group samples; pink treatment group. Each row represented a single gene: green, down-regulated DEGs; red, up-regulated DEGs. The depth of the color denoted the change degree.

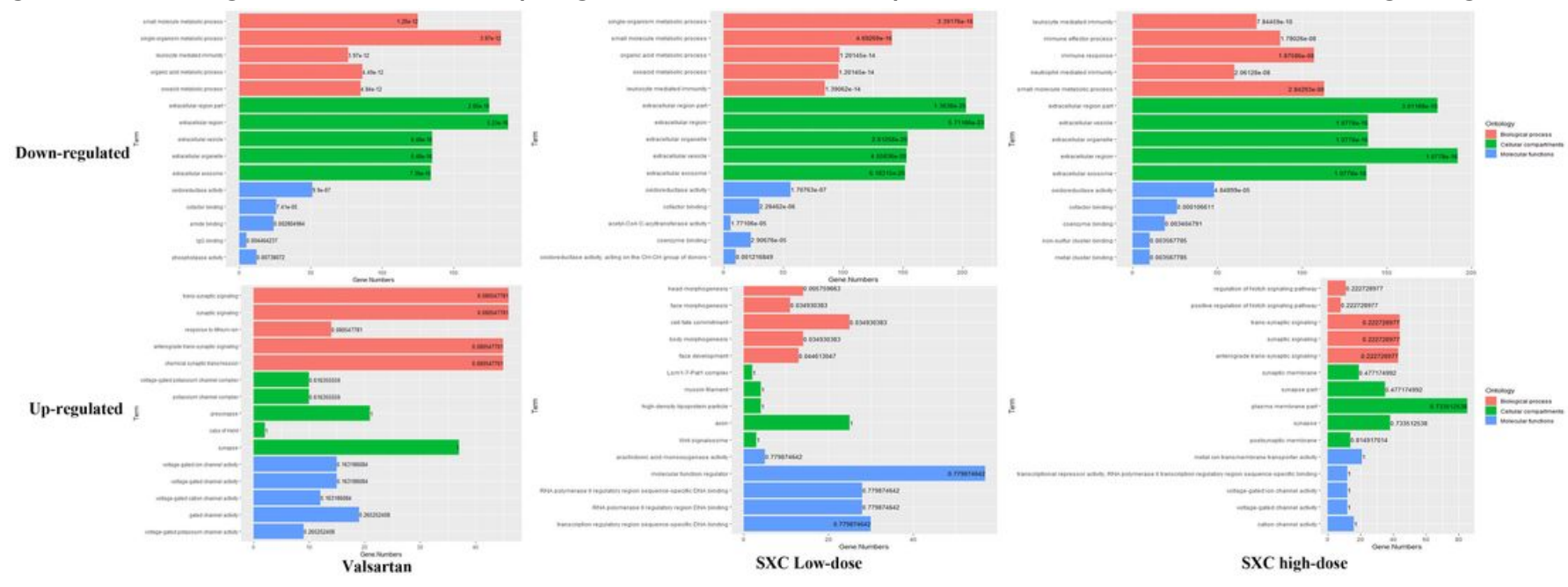

\section{Figure 4}

Significant enriched GO terms of the DEGs. The vertical iterms were the name of the name of the GO terms. The horizontal and the length of the graph represented the gene numbers. The color in the graph denoted the different GO categories: blue, molecular functions; green, cellular compartments; peach, biological process. The number on the bar chart are adjusted $\mathrm{P}$ values. 


\begin{tabular}{|c|c|c|c|c|}
\hline Treatment & Down or up regulated & Clarivate pathway & $P$ value & Adjusted P value \\
\hline \multirow{10}{*}{ Vals artan } & \multirow{5}{*}{ Down regulated } & Regulation of lipid metabolism_PPAR regulation of lipid metabolism & $7.42 \mathrm{E}-08$ & $5.55 \mathrm{E}-05$ \\
\hline & & $\begin{array}{l}\text { Putative pathways of activation of clas sical complement systemin major } \\
\text { depressive disorder }\end{array}$ & $7.42 \mathrm{E}-08$ & $5.55 \mathrm{E}-05$ \\
\hline & & $\begin{array}{l}\text { Pioglitazone and Rosiglitazone in treatment of type } 2 \text { diabetes and } \\
\text { metabolic syndrome X }\end{array}$ & $1.15 \mathrm{E}-07$ & $5.72 \mathrm{E}-05$ \\
\hline & & Immme response_Classical complement pathway & $9.80 \mathrm{E}-07$ & 0.00036661 \\
\hline & & Complement pathway disnuption in thrombotic microangiopathy & $1.57 \mathrm{E}-06$ & 0.000469193 \\
\hline & \multirow{5}{*}{ Up regulated } & Stemcells_BMP signaling in cardiac myogenesis & 0.000427793 & 0.639978705 \\
\hline & & Cell adhesion_Tight junctions & 0.001528795 & 1 \\
\hline & & Regulation of IGF family activity in colorectal cancer & 0.003313826 & 1 \\
\hline & & Hedgehog signaling in breast cancer & 0.003864775 & 1 \\
\hline & & $\begin{array}{l}\text { Notch signaling in oligodendrocyte precurs or cell differentiation in } \\
\text { multiple sclerosis }\end{array}$ & 0.005146142 & 1 \\
\hline \multirow{10}{*}{ SXC low-dose } & \multirow{5}{*}{ Down regulated } & $\begin{array}{l}\text { Pioglitazone and Rosiglitazone in treatment of type } 2 \text { diabetes and } \\
\text { metabolic syndrome } \mathrm{X}\end{array}$ & 3.05E-07 & 0.000394509 \\
\hline & & $\begin{array}{l}\text { Chemokines in inflammation in adipose tissue and liver in obesity, type } \\
2 \text { diabetes and metabolic syndrome } \mathrm{X}\end{array}$ & $5.27 \mathrm{E}-07$ & 0.000394509 \\
\hline & & Regulation of lipid metabolism_PPAR regulation of lipid metabolism & $2.77 \mathrm{E}-06$ & 0.001348889 \\
\hline & & Role of adipose tissue hypoxia in obesity and type 2 diabetes & $3.61 \mathrm{E}-06$ & 0.001348889 \\
\hline & & Leucine, is oleucine and valine metabolismp. 2 & $5.67 \mathrm{E}-06$ & 0.001696466 \\
\hline & \multirow{5}{*}{ Up regulated } & Resistance of pancreatic cancer cells to death receptor signaling & 0.000458545 & 0.665707515 \\
\hline & & Role of osteoblasts in bone lesions formation in multiple myelom & 0.001006162 & 0.665707515 \\
\hline & & Cell adhesion_Tight junctions & 0.001334975 & 0.665707515 \\
\hline & & $\begin{array}{l}\text { Muscle contraction_Delta-type opioid receptor in smoth muscle } \\
\text { contraction }\end{array}$ & 0.003615442 & 1 \\
\hline & & $\mathrm{HCV}$-dependent transcription regulation leading to $\mathrm{HCC}$ & 0.004115694 & 1 \\
\hline \multirow{10}{*}{ SXC high-dose } & \multirow{5}{*}{ Down regulated } & Leucine, is oleucine and valine metabolismp. 2 & $1.41 \mathrm{E}-07$ & 0.00021042 \\
\hline & & Immune response_Classical complement pathway & $7.35 \mathrm{E}-06$ & 0.004918229 \\
\hline & & $\begin{array}{l}\text { Stemcells_Role of Ceramide 1-phosphate, Sphingosine 1-phosphate } \\
\text { and Complement cascade in hematopoietic stemcell horing }\end{array}$ & $1.15 \mathrm{E}-05$ & 0.004918229 \\
\hline & & $\begin{array}{l}\text { Putative pathways of activation of classical complement systemin major } \\
\text { depressive disorder }\end{array}$ & $1.32 \mathrm{E}-05$ & 0.004918229 \\
\hline & & Role of adipose tissue hypoxia in obesity and type 2 diabetes & 0.000109338 & 0.032713792 \\
\hline & \multirow{5}{*}{ Up regulated } & Stemcells_BMP signaling in cardiac myogenesis & 0.002030255 & 1 \\
\hline & & Nociception_Nociceptin receptor signaling & 0.002819131 & 1 \\
\hline & & Role of activation of WNT signaling in the progression of lung cancer & 0.00455593 & 1 \\
\hline & & $\begin{array}{l}\text { Stemcells_Role of BMP signaling in embryonic stemcell neural } \\
\text { differentiation }\end{array}$ & 0.006193407 & 1 \\
\hline & & Tinnitus-associated changes in auditory pathway & 0.00757341 & 1 \\
\hline
\end{tabular}

\section{Figure 5}

Identification of pathways dysregulated in valsartan, SXC low-dose and SXC high-dose treatment. The top 5 pathways most enriched for DEGs in vassartan, SXC low-dose and SXC high-dose treatment group compared to control as measured by microarray. $\mathrm{P}$ value and adjusted $\mathrm{P}$ value were shown in right column. 


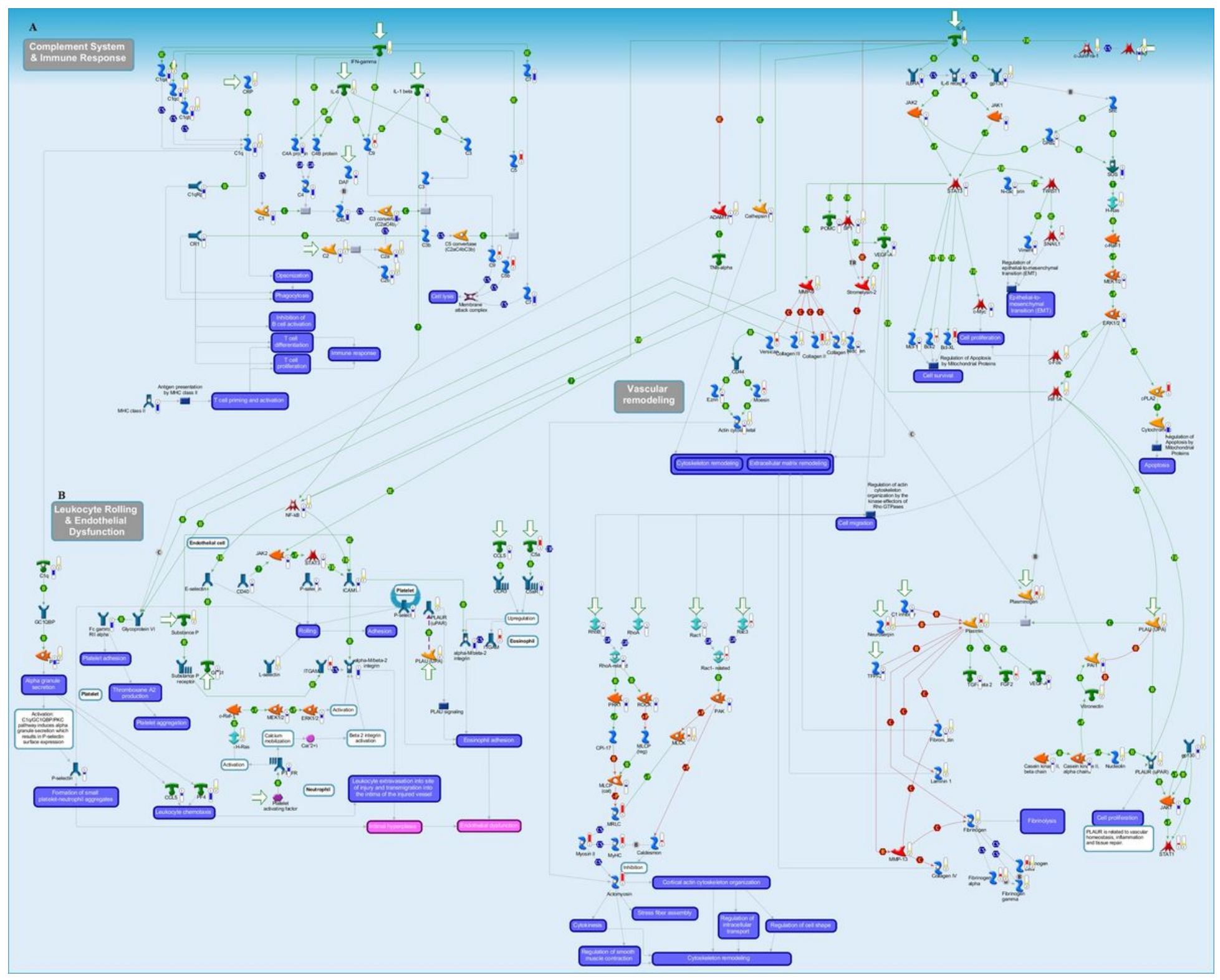

\section{Figure 6}

Causal network model for SXC high-dose treatment compared to control. (A) Complement system, immune response, leukocyte rolling and vascular remodeling processes of the proposed causal net work for SXC high-dose VS. Control. (B) Lipid metabolism, dyslipidemia and inflammatory processes of the proposed causal network model for TCM high dose VS. Control. Blue thermometers indicate downregulated genes while red are upregulated genes. Thermometer numbers represent dataset: 1 , differentially expressed genes; 2 , toplogically significant genes (show in yellow). 


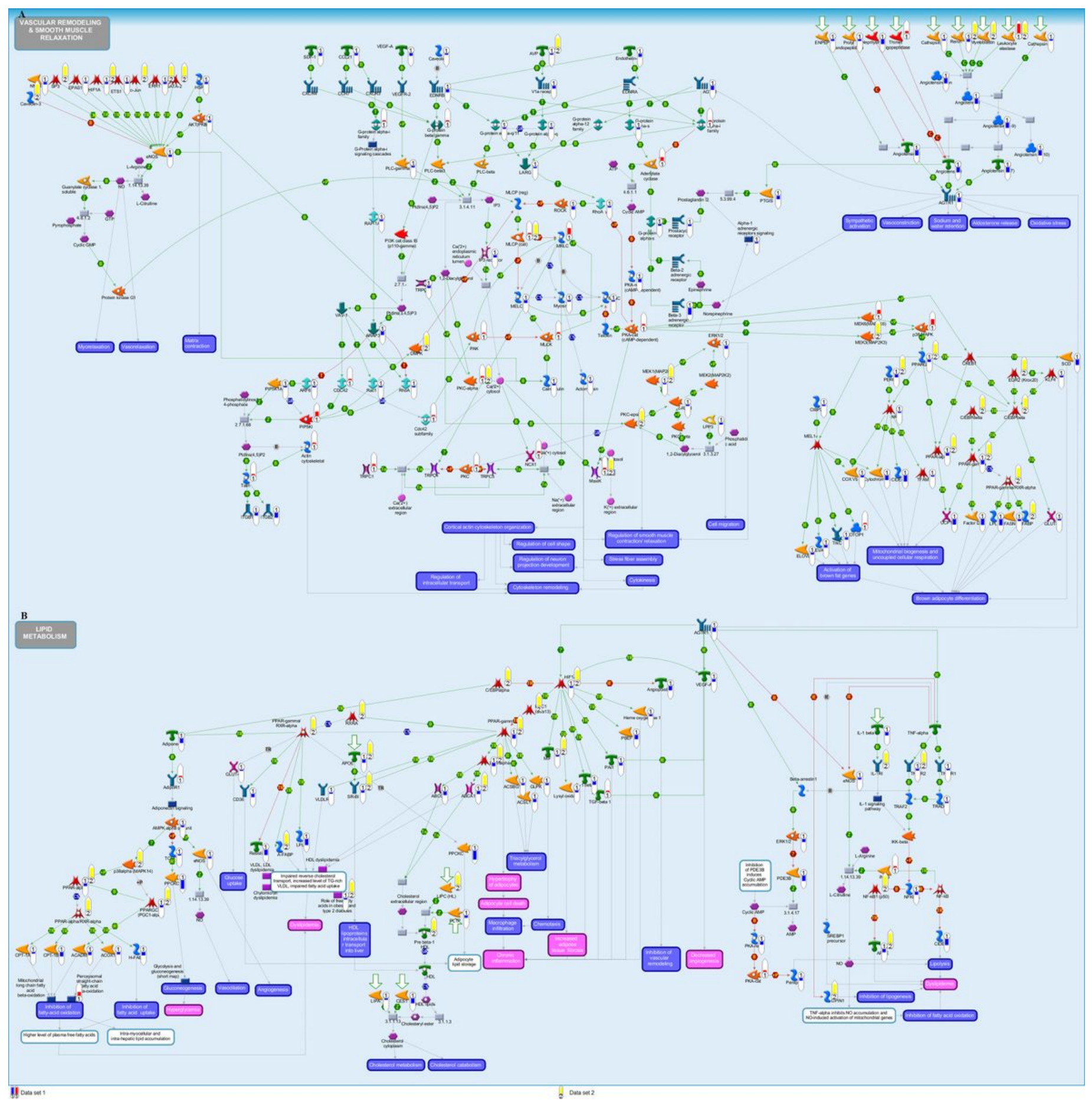

Figure 7

Causal network model for valsartan treatment compared to control. (A) Vascular remodeling and smooth muscle relaxation processes of the proposed causal network model for valsartan VS. Control. (B) Lipid metabolism processes of the proposed causal network model model for valsartan VS. Control. Blue thermometers indicate downregulated genes while red are upregulated genes. Thermometer numbers represent dataset: 1 , differentially expressed genes; 2 , toplogically significant genes (show in yellow).

\section{Supplementary Files}


This is a list of supplementary files associated with this preprint. Click to download.

- S3TableSXChighdoseVsControl.xIsx

- BCARD1900769Checklist.pdf

- S2TableSXClowdoseVsControl.xlsx

- S1TablevalsartanVsControl.xlsx 